MSUCL-1251

\title{
Surface Symmetry Energy
}

\author{
Paweł Danielewicz* \\ National Superconducting Cyclotron Laboratory and \\ Department of Physics and Astronomy, Michigan State University, \\ East Lansing, Michigan 48824, USA
}

(October 25, 2018)

\begin{abstract}
Binding energy of symmetric nuclear matter can be accessed straightforwardly with the textbook mass-formula and a sample of nuclear masses. We show that, with a minimally modified formula (along the lines of the droplet model), the symmetry energy of nuclear matter can be accessed nearly as easily. Elementary considerations for a macroscopic nucleus show that the surface tension needs to depend on asymmetry. That dependence modifies the surface energy and implies the emergence of asymmetry skin. In the mass formula, the volume and surface and (a)symmetry energies combine as energies of two connected capacitors, with the volume and surface capacitances proportional to the volume and area, respectively. The net asymmetry partitions itself into volume and surface contributions in proportion to the capacitances. A combination of data on skin sizes and masses constrains the volume symmetry parameter to $27 \mathrm{MeV} \lesssim \alpha \lesssim 31 \mathrm{MeV}$ and the volume-to-surface symmetry-parameter ratio to $2.0 \lesssim \alpha / \beta \lesssim 2$.8. In Thomas-Fermi theory, the surface asymmetry-capacitance stems from a drop of the symmetry energy per nucleon $S$ with density. We establish limits on the drop at half of normal density, to $0.57 \lesssim S\left(\rho_{0} / 2\right) / S\left(\rho_{0}\right) \lesssim 0.83$. In considering the feeding of surface by an asymmetry flux from interior, we obtain a universal condition for the collective asymmetry oscillations, in terms of the asymmetry-capacitance ratio.
\end{abstract}

Keywords: binding energy formula, symmetry energy, surface symmetry energy, nuclear skin, giant resonance

PACS numbers: 21.10.Dr, 21.10.Gv, 21.60.Ev, 21.65.+f

Typeset using REVTEX

*e-mail: danielewicz@nscl.msu.edu 


\section{INTRODUCTION}

Changes in nuclear properties and in reaction dynamics under the changing nucleon content of a system attract attention in the context of exotic nuclear beams [1]. Determination of the density dependence of the symmetry energy, describing rise in the nuclear energy with an increasing asymmetry between neutrons and protons, could permit extrapolations to the limit of neutron matter and determinations of neutron-star properties [1-3]. Central nuclear reactions can provide varying nuclear densities [1] but constraints on the symmetry energy from structure, pertaining to normal and subnormal densities, are also very important. As an example, the nuclei with a significant excess of neutrons over protons tend to be characterized by larger radii for the neutron than proton distributions, i.e. exhibit neutron skins. It has been noted, within nuclear mean-field calculations [4-7], that the skin size could yield information on the derivative of symmetry energy with respect to density. For knowledge to be easily transferrable between the structure and the reactions, which bring in their own complexity, it is important to understand the role of symmetry energy in the structure at a basic level. For a utility in the reactions, importantly, any quantitative conclusions from the structure cannot be just tied to a specific structure model but need to pertain to universal properties of the medium, even if coarsely.

Most elementary information on nuclear properties stems from the semiempirical energy formula describing the average changes in nuclear binding energy with the nucleon content $[8,9]$. The volume and surface terms in the standard formula pertain to symmetric systems. The volume coefficient provides the binding energy per nucleon in the symmetric nuclear matter; the surface coefficient, up to a factor, provides the surface tension. Notably, the symmetry term in the standard energy formula has a volume character only. However, as we point out in this paper, basic intrinsic consistency of the semiempirical formula requires the inclusion of a surface symmetry term. Quantitatively, such a term turns out further to be essential for describing energies of light asymmetric nuclei. Moreover, inclusion of such a term is essential for drawing conclusions about the symmetry energy in general, from the nuclear structure. The surface symmetry energy appears in the literature in advanced energy formulas, generally in two different type of forms, in an expanded form such as in the liquid drop model by Myers and Swiatecki [10-13] or in more complex forms of the liquid droplet model by the above authors [14-16,12]. Both formulations are currently employed in the descriptions of nuclei $[12,13,17]$.

In this work we follow a general type of considerations for the energy of a nucleus treated as a binary macroscopic system with a surface. Similar type of considerations can apply to other binary systems with an interface, such as between two phases [18]. We show that, when the surface energy is affected by the particle asymmetry within the system, then the thermodynamic consistency requires that some of the asymmetry moves to the surface, i.e. an asymmetry skin develops. Minimization of the net nuclear energy with respect to the partition of asymmetry produces an expression for the symmetry energy such as in the more recent versions of the droplet model $[15,16]$. (In the droplet model, the skin size is a basic parameter and, as such, one of the starting points.) We show that the potentially confusing expression for the energy is easily comprehended using a capacitor analogy. The symmetry energy, quadratic in the asymmetry, can be interpreted in terms of the energy of a capacitor

for asymmetry, with the capacitance for asymmetry proportional to the nuclear volume in 
the standard energy formula [8,9]. However, in the actual nuclei, the surface provides an additional capacitance for the asymmetry, proportional to the surface area. In the end, the net symmetry energy is given in terms of the net asymmetry squared divided by the net capacitance, where one term in the capacitance grows as the volume and the other as the area. Notably, the liquid-drop type formula follows when the surface capacitance is small compared to the volume capacitance. In general, when ignoring the surface term in the nuclear energy formula, the whole asymmetry capacitance is attributed to the interior and, thus, the volume capacitance is overestimated which is equivalent to underestimating the volume symmetry coefficient.

On adding a single parameter to the textbook nuclear energy formula, representing the surface symmetry energy, we fit the measured ground-state energies [19] to establish limits on the symmetry energy coefficients. Notably, the tradition within the description of nuclear energies in terms of advanced formulas [10-16,12], often supplemented with structure calculations, has been to quote optimal or preferred values for utilized constants. While this is fine as far as parameters of a model are concerned, this is not sufficient when the fundamental matter properties are being sought. As a drastic example, the authors of [12] produce parameters in two different descriptions of nuclear energies that yield quite different surface symmetry properties for nuclear matter, seemingly without a concern. For the fundamental properties, the validity of employed theory needs to be assessed and, importantly, the features of the optimization, i.e. the value for the property needs to be quoted together with an uncertainty. However, this is normally not done in the context of advanced mass formulas. Obviously, if the fit minimum is very flat, or nearly degenerate, the property is not well constrained. One plain example of how things can go wrong with a fit is when a parameter is included that has little bearing on the quality of the fit, yet the optimization produces a value for the parameter. For validity of conclusions, the importance of the specific parameter for the fit needs to be demonstrated. For a multitude of parameters, the issues of fit stability may need to be explored.

In analyzing our fit, we demonstrate that the surface symmetry term is required, in order to describe the energies of light asymmetric nuclei at the level similar to the other nuclei. However, once the surface symmetry term is included, the minimum for the fit to masses is relatively flat when the surface and volume symmetry coefficients are varied along a correlation valley enforced by the need to reproduce, for heavy nuclei, the magnitude of symmetry coefficient in the standard formula.

We subsequently turn to the analysis of the size of asymmetry skins. The dependence of the size on net nuclear asymmetry and on mass probes the ratio of volume to surface symmetry coefficients. This is because the size represents the surface asymmetry charge which, in relation to the net asymmetry charge, tests the ratio of surface to volume capacitances. The dependence of skin sizes on the difference of proton-neutron separation energies probes, on the other hand, the surface energy coefficient. This is because, in the capacitor analogy, the difference of separation energies represents the voltage and the dependence of surface asymmetry charge on voltage tests the surface capacitance. On analyzing a variety of data on skin sizes [20-27], upon inclusion of Coulomb corrections, we obtain constraints that, in combination with the constraints from mass analysis, produce a relatively narrow correlation range for the volume and surface symmetry coefficients.

In illustrating further the importance of the surface symmetry energy for nuclei, we de- 
rive new solutions for asymmetry oscillations in nuclei, with the asymmetry flux feeding the surface. This extends the treatment of isoscalar modes where the internal compression and the surface deformation are coupled [28]. We obtain a boundary condition of a simple universal form, selecting wavenumbers for different multipolarities of vibrations. We find that the use of a realistic surface symmetry coefficient improves significantly the description of the dependence of giant dipole resonance energy on mass, as compared to the use of assumption that the surface accepts no asymmetry. The mode of oscillation, in our description, changes from one that is closer to the Goldhaber-Teller (i.e. surface) type [29] to one closer to the Steinwedel-Jensen (i.e. volume) type [30] as the nuclear mass increases.

As a final step, we analyze the nature of the surface symmetry energy at a microscopic level within the extended Thomas-Fermi model. We show that an extra capacitance for the asymmetry associated with the surface is due to the drop of symmetry energy per nucleon with density (lower energy penalty $\equiv$ higher capacitance). If the symmetry energy per nucleon is density independent, no extra surface capacitance emerges. Within the ThomasFermi approximation, for a given density profile of symmetric matter, the ratio of the volume to surface energy parameters is solely related to the shape of the density dependence of symmetry energy. Equipped with this knowledge, we turn to an analysis of the mean-field structure calculations in the literature [5-7], where numerical experimentation was used to find a relation between the skin sizes and features of the nuclear equation of state. The combination of our earlier results and those in the Thomas-Fermi approximation indicates that the skin for a given nucleus is primarily related to the shape of the density dependence of symmetry energy per nucleon. Using the results from the structure calculations [7], we are able to narrow the bracket on the density dependence of symmetry energy by nearly a factor of 2 compared to what was done before [7]. Also, within the Thomas-Fermi approximation, we cross-test the validity of our general results for skins.

The paper is organized as follows. In the next section, we discuss the symmetry energy within the binding-energy formula and we analyze known masses in terms of the modified formula, to constrain the symmetry energy parameters. In Sec. III, we derive expressions

for the nuclear skin size in terms of the symmetry energy parameters, as a function of mass and charge numbers and as a function of separation energies. We analyze data in terms of obtained expressions to get additional constraints on the symmetry parameters, narrowing the range allowed by data. The asymmetry oscillations are discussed in Sec. IV. The constraints on the low-density behavior of symmetry energy are discussed in Sec. V. Our results are recapitulated in Sec. VI.

\section{BINDING ENERGY FORMULA}

\section{A. Elementary Considerations}

We start out with a reminder of elementary issues. The Weizsäcker nuclear energy formula $[8,9]$

$$
E=-a_{V} A+a_{S} A^{2 / 3}+a_{a} \frac{(N-Z)^{2}}{A}+a_{C} \frac{Z(Z-1)}{A^{1 / 3}}
$$


separates out different contributions to the nuclear energy. The r.h.s. negative volumeterm represents the energy in the bulk limit of symmetric nuclear-matter in the absence of Coulomb interactions. Proportionality to the mass number $A$ for this largest in magnitude term, together with an approximately constant nucleon density of $\rho_{0}=3 /\left(4 \pi r_{0}^{3}\right) \simeq$ $0.160 \mathrm{fm}^{-3}$ in the nuclear interiors, underscore the short-range nature of nuclear interactions. The three subsequent terms in the Weizsäcker formula represent an increase in the energy compared to the bulk symmetric limit. These terms are associated, respectively, with the size of the surface, with an asymmetry in the number of protons $Z$ and neutrons $N$ and with the Coulomb repulsion. Regarding the (a)symmetry term in the Weizsäcker formula, we observe that this term scales like $A$ when the nucleon number is changed and that we do not have a surface (a)symmetry energy term, scaling like $A^{2 / 3}$. The $N-Z$ asymmetry reduces the binding because it enhances the role of Pauli principle and because the likenucleon interactions are less attractive than the neutron-proton interactions. The precise symmetry of that term around $N-Z=0$ expresses the symmetry of nuclear forces under the neutron-proton exchange, or charge symmetry.

With the radius $r_{0}$ of the nuclear volume per nucleon, the size of the nuclear surface is $\mathcal{S}=4 \pi r_{0}^{2} A^{2 / 3}$, and we can write the surface energy as

$$
E_{S}=a_{S} A^{2 / 3}=\frac{a_{S}}{4 \pi r_{0}^{2}} \mathcal{S}
$$

The tension $\sigma$ is the derivative of energy with respect to surface area:

$$
\sigma=\frac{\partial E}{\partial \mathcal{S}}=\frac{a_{S}}{4 \pi r_{0}^{2}}
$$

The tension represents a work per unit area needed to create the surface. The work is required because the nucleons at the surface are less bound than in the interior. However, we know that the $N-Z$ asymmetry reduces the interior binding. Thus, with an increasing asymmetry, the work required per unit surface, the tension, should drop. Coulomb effects will also affect the surface, but will be dealt with later.

As a microscopic quantity, the tension should depend on a microscopic quantity associated with the particle asymmetry. The respective quantity common for different subsystems in contact is the chemical potential. With the nucleon chemical potentials given by

$$
\mu_{n}=\frac{\partial E}{\partial N} \quad \text { and } \quad \mu_{p}=\frac{\partial E}{\partial Z}
$$

the potential conjugate to the asymmetry $N-Z$ is half of the the nucleon potential difference, $\mu_{a}=\left(\mu_{n}-\mu_{p}\right) / 2$, and the change in energy associated with the particle numbers is:

$$
d E=\mu_{n} d N+\mu_{p} d Z=\mu d A+\mu_{a} d(N-Z),
$$

with the median chemical potential $\mu=\left(\mu_{n}+\mu_{p}\right) / 2$.

Given that the tension should drop no matter, under the charge symmetry, whether a neutron or proton excess develops, the tension needs to be, in the lowest order, quadratic in the asymmetry potential $\mu_{a}$ :

$$
\sigma=\sigma_{0}-\gamma \mu_{a}^{2}
$$


where $\gamma>0$. As the tension is a derivative of the surface energy, the dependence of $\sigma$ on $\mu_{a}$ implies the dependence of the surface energy on $\mu_{a}$, with serious consequences.

To demonstrate these consequences, let us construct the thermodynamic potential generated by a Legendre transformation from the asymmetry $N-Z$ to the potential $\mu_{a}$ :

$$
\Phi=\mu_{a}(N-Z)-E
$$

For definiteness, we next consider changes in the net system when the asymmetry changes, while $A$ and shape remain fixed. The derivative of $\Phi$ with respect to $\mu_{a}$ gives the net asymmetry for the nucleus $N-Z$ :

$$
\frac{\partial \Phi}{\partial \mu_{a}}=N-Z+\mu_{a} \frac{\partial(N-Z)}{\partial \mu_{a}}-\frac{\partial E}{\partial \mu_{a}}=N-Z,
$$

cf. (5). On the r.h.s. of (7), we can separate the energy $E$ into the surface $E_{S}$ and volume $E_{V}$ contributions, $E=E_{S}+E_{V}$. When the asymmetry changes, the change in volume energy is $d E_{V}=\mu_{a} d\left(N_{V}-Z_{V}\right)$, where $d\left(N_{V}-Z_{V}\right)$ is the change in particle asymmetry within the nuclear volume, expected equal to $d(N-Z)$. If we now substitute (7) into the l.h.s. of (8) and compare to the r.h.s. there, we find

$$
\mu_{a}\left(\frac{\partial(N-Z)}{\partial \mu_{a}}-\frac{\partial\left(N_{V}-Z_{V}\right)}{\partial \mu_{a}}\right)-\frac{\partial E_{S}}{\partial \mu_{a}}=0 .
$$

Here we can see, though, that we will run into a contradiction if we identify changes in the net nuclear asymmetry $N-Z$ with the changes in the interior asymmetry $N_{V}-Z_{V}$, when the surface energy $E_{S}$ depends on chemical potential $\mu_{a}$. The net and interior asymmetries must be different, and the difference $N_{S}-Z_{S}=N-Z-\left(N_{V}-Z_{V}\right)$ must, as a consequence, reside within the nuclear surface. To tackle the conceptual issue of how a surface can contain a particle number, we must consider how the surface quantities get to be defined.

For an interface between phases in equilibrium, Gibbs [18] suggested to take a small but macroscopic volume surrounding an element of the interface and, besides the actual system, consider an idealized reference system, cf. Fig. 1. In the idealized system, the densities of thermodynamic quantities, such as the particle density, change abruptly at the interface from a value characterizing one of the phases away from the interface to the value for the other phase. The surface share $F_{S}$ for the surface element $\mathcal{S}$ is then the difference between the value of the quantity for an actual system $F$ and the value for an idealized system $F_{i d}$ : $F_{S}=F-F_{i d}$. In terms of densities, for the case of a nucleus in equilibrium with the vacuum, this further yields for a quantity $F: F_{S}=\mathrm{f} \mathcal{S}=F-V f_{V}$, where $f_{V}$ is the density of $F$ characteristic for the interior and $f$ represents the surface density. The problem is, however, that the result for the surface depends on a precise position assumed for the interface, and a condition may be imposed further to specify the position. For a nucleus it is natural to assume that the surface contains no mass number and, for a spherical nucleus, this is equivalent to taking the equivalent sharp-sphere radius for the surface of $R=r_{0} A^{1 / 3}$.

Only one condition related to the particle numbers, however, suffices to localize the surface. In a two-component system, the attempts to localize the surface using one of the components or the other would lead to two different positions. If the surface is localized using the net particle density, a finite surface asymmetry $N_{S}-Z_{S}$ results, even though the 
surface has a vanishing net nucleon number $A_{S}=N_{S}+Z_{S}=0$. For a spherical nucleus, this corresponds to having two different equivalent sharp-sphere radii for neutrons $R_{n}$ and protons $R_{p}$, bracketing the sharp-sphere radius for the matter $R$, cf. Fig. 2 .

The Gibbs prescription can be used for clarifications, but also as a direct tool, as e.g. later illustrated by a consideration in Subsection V A or when pursuing curvature effects. We next relate the surface asymmetry excess to the asymmetry potential. Either by following the Gibbs prescription, with a small volume surrounding some surface element, or by isolating contributions pertaining to the surface element in the energy considerations before, we find

$$
d E_{S}=\sigma d \mathcal{S}+\mu_{a} d\left(N_{S}-Z_{S}\right)
$$

under independent variations of asymmetry and of the surface element size. From changes in $E_{S}$, when taking an increasing fraction of the net surface, we find that

$$
E_{S}=\sigma \mathcal{S}+\mu_{a}\left(N_{S}-Z_{S}\right)
$$

On taking a differential of the above, with the result

$$
d E_{S}=\mathcal{S} d \sigma+\sigma d \mathcal{S}+\left(N_{S}-Z_{S}\right) d \mu_{a}+\mu_{a} d\left(N_{S}-Z_{S}\right)
$$

and on subtracting from this result the relation (10), side by side, we obtain a Gibbs-Duhem type relation. That last relation yields

$$
\frac{N_{S}-Z_{S}}{\mathcal{S}}=-\frac{d \sigma}{d \mu_{a}}=2 \gamma \mu_{a}
$$

where, at the end, we employed Eq. (6).

With (13) and (6), we get from (11):

$$
E_{S}=\sigma_{0} \mathcal{S}+\gamma \mu_{a}^{2} \mathcal{S}=E_{S}^{0}+\frac{1}{4 \gamma} \frac{\left(N_{S}-Z_{S}\right)^{2}}{\mathcal{S}},
$$

where $E_{S}^{0}$ is the surface energy in absence of the surface asymmetry. At this point, we may cross-check the consistency of our result (14) with its derivation. Thus, from (14), we find

$$
\begin{aligned}
d E_{S} & =d E_{S}^{0}+\frac{1}{4 \gamma} \frac{2\left(N_{S}-Z_{S}\right)}{\mathcal{S}} d\left(N_{S}-Z_{S}\right)-\frac{1}{4 \gamma} \frac{\left(N_{S}-Z_{S}\right)^{2}}{\mathcal{S}^{2}} d \mathcal{S} \\
& =\left(\sigma_{0}-\gamma \mu_{a}^{2}\right) d \mathcal{S}+\mu_{a} d\left(N_{S}-Z_{S}\right)=\sigma d \mathcal{S}+\mu_{a} d\left(N_{S}-Z_{S}\right),
\end{aligned}
$$

which agrees with (10) and where we utilized (13) and $d E_{S}^{0}=\sigma_{0} d \mathcal{S}$. In connection with (14), we may note that although the surface tension decreases with the asymmetry, Eq. (6), the net surface energy increases with the asymmetry.

On account of charge symmetry for the interior nuclear energy per unit volume, to lowest order in the asymmetry, the volume energy will have the form

$$
E_{V}=E_{V}^{0}+V \alpha \rho_{0}\left(\frac{N_{V}-Z_{V}}{A}\right)^{2}=E_{V}^{0}+\alpha \frac{\left(N_{V}-Z_{V}\right)^{2}}{A},
$$

where we factorized the coefficient in the energy density so as to get the net volume energy in conformance with (1) and where $E_{V}^{0}\left(\equiv a_{V} A\right)$ is the energy for a symmetric system. 
For an analogy with (16), we introduce a constant with the dimension of energy in $E_{S}$, $\beta=1 /\left(16 \pi r_{0}^{2} \gamma\right)$, to write the r.h.s. of (14) as

$$
E_{S}=E_{S}^{0}+\beta \frac{\left(N_{S}-Z_{S}\right)^{2}}{A^{2 / 3}} .
$$

In the volume energy we use a constant $\alpha$ in place of $a_{a}$ of (1) to make a distinction between the symmetry constant in the bulk and the constant in use in the binding-energy formula. As will be next seen, $a_{a}$ in use generally results from a combination of $\alpha$ and $\beta$.

Minimization of the net system energy $E$ under the constraint of net asymmetry,

$$
E=E_{V}+E_{S} \quad \text { with } \quad N-Z=N_{V}-Z_{V}+N_{S}-Z_{S},
$$

yields the condition of equal chemical asymmetry potentials for the interior and surface,

$$
\begin{aligned}
0 & =\frac{\partial E}{\partial\left(N_{V}-Z_{V}\right)}=\frac{\partial E_{V}}{\partial\left(N_{V}-Z_{V}\right)}-\frac{\partial E_{S}}{\partial\left(N_{S}-Z_{S}\right)} \\
& =2 \alpha \frac{N_{V}-Z_{V}}{A}-2 \beta \frac{N_{S}-Z_{S}}{A^{2 / 3}} .
\end{aligned}
$$

With this, we find for the asymmetry ratios

$$
\frac{N_{S}-Z_{S}}{N_{V}-Z_{V}}=\frac{\alpha}{\beta A^{1 / 3}}, \quad \text { and } \quad \frac{N_{S}-Z_{S}}{N-Z}=\frac{\frac{N_{S}-Z_{S}}{N_{V}-Z_{V}}}{1+\frac{N_{S}-Z_{S}}{N_{V}-Z_{V}}}=\frac{1}{1+\frac{\beta}{\alpha} A^{1 / 3}} .
$$

The net energy then becomes equal to

$$
\begin{aligned}
E & =E_{V}+E_{S}=E_{0}+\frac{\alpha}{A} \frac{(N-Z)^{2}}{\left(1+\frac{\alpha}{\beta} A^{-1 / 3}\right)^{2}}+\frac{\beta}{A^{2 / 3}} \frac{(N-Z)^{2}}{\left(1+\frac{\beta}{\alpha} A^{1 / 3}\right)^{2}} \\
& =E_{0}+\frac{\alpha}{1+\frac{\alpha}{\beta} A^{-1 / 3}} \frac{(N-Z)^{2}}{A} .
\end{aligned}
$$

\section{B. Capacitors for Asymmetry}

If a problem can be mapped onto one that is well understood, existing intuitions can be exploited to make predictions and lay out results with little need for any derivations, and this pertains to the last results. The quadratic energies (16) and (17), with the constraint (18), invite e.g. an analogy to two connected springs, of spring constants $\frac{\alpha}{2 A}$ and $\frac{\beta}{2 A^{2 / 3}}$, respectively, stretched between separated supports. The context, however, suggests an even more appropriate analogy to two connected capacitors carrying charges $q_{X}=N_{X}-Z_{X}$ $(X=V, S)$, of capacitances $C_{V}=\frac{A}{2 \alpha}$ and $C_{S}=\frac{A^{2 / 3}}{2 \beta}$, respectively, to yield capacitor energies

$E_{X}=E_{X}^{0}+\frac{q_{X}^{2}}{2 C_{X}}$. As the asymmetry moves in-between the capacitors, the equilibrium situation is that of a charge in proportion to capacitance, yielding

$$
\frac{N_{S}-Z_{S}}{N_{V}-Z_{V}}=\frac{C_{S}}{C_{V}}=\frac{\alpha}{\beta A^{1 / 3}} \quad \text { and } \quad \frac{N_{S}-Z_{S}}{N-Z}=\frac{C_{S}}{C_{V}+C_{S}}=\frac{1}{1+\frac{\beta}{\alpha} A^{1 / 3}} .
$$


The net capacitor energy, finally, is given in terms of the net charge and net capacitance

$$
E=E^{0}+\frac{q^{2}}{2\left(C_{V}+C_{S}\right)}=E^{0}+\frac{(N-Z)^{2}}{\frac{A}{\alpha}+\frac{A^{2 / 3}}{\beta}}=E_{0}+\frac{\alpha}{1+\frac{\alpha}{\beta} A^{-1 / 3}} \frac{(N-Z)^{2}}{A} .
$$

The connected capacitor and the nuclear symmetry energy problems are, at this level, completely equivalent.

The symmetry energy of (23) should, generally, replace that in the energy formula (1), with $\beta \rightarrow \infty(\gamma \rightarrow 0$, in (6)) obviously corresponding to the standard formula. In (22), we observe that the surface fraction of the asymmetry excess decreases as nuclear mass increases, because the surface capacitance grows only as $A^{2 / 3}$. It may be tempting to expand the symmetry energy (23) in the surface-to-volume capacitance fraction. However, whether

this can be done depends on the volume-to-surface coefficient ratio $\alpha / \beta$. For large enough ratios and small $A$, we, in fact, principally might be able to ignore 1 in the denominator and get the symmetry energy such as characteristic for the surface storage of asymmetry. (This would express the fact that, just as the volume capacitance grows faster than surface capacitance, it also drops faster.) Later in this and in the following section we shall see what $\alpha / \beta$ values are favored by data.

\section{Coulomb Effects}

So far, we have not considered the effect of Coulomb interactions on the surface asymmetry and, generally, the interplay of Coulomb and symmetry energies in the net energy. The Coulomb energy may be represented as

$$
\begin{aligned}
E_{C} & =E_{V}^{C}+E_{V S}^{C}+E_{S}^{C}=\frac{e^{2}}{4 \pi \epsilon_{0}} \frac{1}{R}\left(\frac{3}{5} Z_{V}^{2}+Z_{V} Z_{S}+\frac{1}{2} Z_{S}^{2}\right) \\
& =\frac{a_{C}}{A^{1 / 3}}\left(Z_{V}^{2}+\frac{5}{3} Z_{V} Z_{S}+\frac{5}{6} Z_{S}^{2}\right)
\end{aligned}
$$

where the three terms correspond to the volume, volume-surface and surface interactions, respectively, and $Z_{V}=Z-Z_{S}$.

The combination of the symmetry energies (16) and (17) and the Coulomb energy (24) is quadratic in the surface excess and is easily found to minimize at:

$$
N_{S}-Z_{S}=\frac{N-Z-\frac{a_{C}}{12 \alpha} Z A^{2 / 3}}{1+\frac{\beta}{\alpha} A^{1 / 3}+\frac{a_{C}}{48 \alpha} A^{2 / 3}} \approx \frac{N-Z-\frac{a_{C}}{12 \alpha} Z A^{2 / 3}}{1+\frac{\beta}{\alpha} A^{1 / 3}} .
$$

The last approximation expresses the fact the Coulomb interactions represent a very soft spring in the energy combination, compared to the volume asymmetry spring. Compared to (22), we see that, as might be expected, the Coulomb interactions try to tilt the surface asymmetry towards a proton excess. Quantitatively, however, the Coulomb correction to the asymmetry excess is quite negligible for light and medium nuclei and acquires only a limited significance for heavy nuclei.

As to the energies, even when the Coulomb correction to the surface asymmetry has a significance, the corresponding changes in the asymmetry energy are quite ignorable. This 
because around the minimum, specified by (22), the changes in the asymmetry energy are quadratic in the asymmetry correction. On the other hand, the correction to the Coulomb energy, from the development of surface asymmetry,

$$
\left.\Delta E_{C} \simeq \frac{\partial E_{C}}{\partial Z_{S}}\right|_{0} Z_{S} \simeq \frac{a_{C} Z^{2}}{A^{1 / 3}} \frac{N-Z}{6 Z} \frac{1}{1+\frac{\beta}{\alpha} A^{1 / 3}}
$$

may have a moderate significance within the net energy and, in heavy nuclei, can reach a comparable magnitude to the correction to the Coulomb energy from surface diffuseness.

\section{Relation to the Droplet Model}

The droplet model [14] utilizes the relative displacement of neutron and proton surfaces as one of the different parameters describing a nucleus. A global energy functional is constructed for a nucleus, incorporating an effect of the surface displacement on the net energy. This functional is minimized with respect to the different parameters in order to arrive at the ground-state energy.

While the droplet model contains a variety of potentially useful details, these can easily shroud the issues of symmetry energy. In fact, our own understanding of that aspect of the model stems largely from a mapping of the results there onto those in the present paper. In particular, if the final result for energy in the droplet model in [15] (and in later papers on the model) is expanded in the net particle asymmetry, then a symmetry energy emerges such as in Eq. (21), provided our constants are identified with those in the droplet model as:

$$
\alpha=J \quad \text { and } \quad \beta=\frac{4}{9} Q=\frac{\frac{4}{9} H}{1-\frac{2}{3} P / J} .
$$

The individual constants $H, P$ and $G$, found equal to $G=3 J P / 2 Q$, describe the assumed dependence [14] of the surface energy on bulk asymmetry and on normalized size of the asymmetry skin. The different definitions of the surface energy within the model are discussed in Refs. [31,32].

If the net energy in the droplet model $[15,12]$ is expanded simultaneously in terms of the net asymmetry and in the Coulomb coefficient $a_{C}$, then a correction to the energy is found in the form such as in (26).

\section{E. Comparison to Binding-Energy Data}

We next examine what can be learnt on the nuclear symmetry energy from measured binding energies [19], $B=-E$, employing the results from above, by following an energy formula with only one more adjustable parameter than in the textbooks [28], to represent the surface symmetry energy. Specifically, we fit the measured energies with

$$
E=-a_{V} A+a_{S} A^{2 / 3}+\frac{\alpha}{1+\frac{\alpha}{\beta} A^{-1 / 3}} \frac{(N-Z)^{2}}{A}+a_{C} \frac{Z(Z-1)}{A^{1 / 3}(1+\Delta)}-\delta .
$$


Compared to (1), we changed the symmetry term to (23) and we added the standard pairing term $\delta= \pm a_{p} A^{-1 / 2}, 0$. We either carry out fits with $\Delta=0$ in the Coulomb term, i.e. the energy formula modified, from the standard, only by the inclusion of surface symmetry energy, or with

$$
\Delta=\frac{5 \pi^{2}}{6} \frac{d^{2}}{r_{0}^{2} A^{2 / 3}}-\frac{1}{1+\frac{\beta}{\alpha} A^{1 / 3}} \frac{N-Z}{6 Z},
$$

including the effects of surface diffuseness and of the asymmetry skin (26) on the Coulomb energy. In the first r.h.s. term above, $d \approx 0.55 \mathrm{fm}$ is the diffuseness parameter in the Fermi function from the parametrization of nuclear charge distributions [33],

$$
\rho(r)=\frac{\rho_{0}}{1+\exp [(r-c) / d]}
$$

that term stems from an expansion of the Coulomb energy for (30) in terms of $d$.

Qualitatively, results from the two fits indicated above are similar but they differ in quantitative details. Obviously, inclusion of the volume and surface competition, in the symmetry energy, changes the dependence of the net energy on asymmetry as a function of mass. However, the Coulomb energy is also a source of the dependence on asymmetry and deciding on the details in one energy contribution depends on the details in the other. We believe that more reliable results are obtained with the finite $\Delta$, Eq. (29), in (28) as we only incorporate information available from electron scattering or following from intrinsic consistency of the considerations. The number of independent parameters in the fit with (29) is the same as in the $\Delta=0$ fit, precluding, in practice, a fit runaway in the parameter space.

In the fits, we optimize the sum of absolute deviations of the theoretical energies from experimental, rather than the sum of the deviations squared, as the intention is to provide an average description of the nuclear features. Results, however, weakly depend on that choice. In the optimization, we include the energies of all nuclei with $A \geq 2$, but exclude the energies deduced from trends [19]. We either let all parameters in (28) vary in an unconstrained manner or we constrain $\alpha / \beta$ or both of the symmetry parameters.

The average per nucleus deviation of the theoretical energy from experimental exhibits a narrow valley in the space of $\alpha / \beta$ vs $\alpha$, as illustrated in Fig. 3 for the fit with (29). The valley is described by the equation $\alpha \approx(21.5+3.1 \alpha / \beta) \mathrm{MeV}$. An analogous valley is found for the fit with $\Delta=0$, but the valley is then shifted by about $10 \%$ to higher values in $\alpha$. The strong correlation may be understood in terms of the need to properly describe energies for the heaviest nuclei with largest asymmetries requiring $\alpha /\left(1+A^{-1 / 3} \alpha / \beta\right) \approx a_{a}$, for $A \sim 200$.

Along the valley, the average deviation minimizes at $\alpha / \beta \sim 1.7$, with the value of $\overline{\left|E-E^{e x p}\right|}=1.97 \mathrm{MeV}$. However, the minimum along the valley is rather flat. To understand the nature of the minimum, we carry out the following procedure. We carry out an optimization of all parameters in the energy formula at a fixed $\alpha / \beta$. Then, keeping all parameters fixed including $\alpha / \beta$ but excluding $\alpha$, we invert the energy formula to deduce $\alpha$ from the mass of an individual nucleus. With this, we test locally the global fit. If the formula provides a proper description, then, on the average, we should get the same $\alpha$ from an individual mass as from the global fit. Notably, for the inversion to be significant, the 
asymmetry should be large and we demand that $|N-Z| / A>0.2$. Sample results are displayed in Fig. 4, as a function of $A$.

First to consider are the results obtained at $\alpha / \beta=0$, i.e. ignoring the surface symmetry energy, as in the textbook mass formulas. Represented by the diamonds in Fig. 4 , the results for $\alpha$ (i.e. $a_{a}$ in this case) group coarsely into a line. At large $A$, the line from symbols oscillates, exhibiting the shell structure, around the value of $\alpha$ from the global fit, represented by a straight line. However, at $A \lesssim 50$ the values from a local inversion systematically drop below $a_{a}$ from the global fit. Such a drop for $a_{a}$ at low $A$ is expected from a competition between the volume and surface symmetry energies (28). If we raise the parameter ratio, such as to the value of $\alpha / \beta=2$, we can get the locally restored values of $\alpha$ to oscillate around the global fit value down to very low $A$. Regarding the restored values of $\alpha$, we may note in Fig. 4 that they locally exhibit less scatter for the fit with (29) than with $\Delta=0$, suggesting that the first fit grasps the physics better. Finally, if we assume a too high value of the parameter ratio, such as $\alpha / \beta=6$, we get the restored $\alpha$ to rise up at low $A$ in an opposite tendency to that for $\alpha / \beta=0$.

The gain in the description of low- $A$ nuclei when using a finite $\alpha / \beta$ can be further appreciated by looking directly at magnitudes of the average fit deviations in the low- $A$ region. Thus, if we employ $\alpha / \beta=0$ in (28) in the global fit with (29), then for nuclei in the region of $5 \leq A \leq 50$ and $|N-Z| / A>0.2$, we get $\overline{\left|E-E^{\exp }\right|}=7.4 \mathrm{MeV}$, far in excess of the average deviation of $2.37 \mathrm{MeV}$ for all nuclei. On the other hand, for $\alpha / \beta \sim 1.7$, we get an average deviation of $\overline{\left|E-E^{e x p}\right|}=2.3 \mathrm{MeV}$ in the region of $5 \leq A \leq 50$ and $|N-Z| / A>0.2$, close to the average deviation of $1.97 \mathrm{MeV}$ for all nuclei.

While, at the lower $A$, the use of an intermediate value of $\alpha / \beta$ improves the performance of the energy formula, at the highest $A$ the separate sensitivity to the ratio and $\alpha$ is quite reduced. With most of the measured nuclei of high anisotropies at the high $A$, the reduction in the deviation between the formula and the experimental energies, when averaged over all $A$, is just $\sim 0.40 \mathrm{MeV}$ per nucleus for the absolute minimum in Fig. 3, compared to the optimal case of $\alpha / \beta=0$. Considering in particular Fig. 4, we need to decide how accurate our phenomenological theory should be in describing the nuclei. While the superiority of the $\alpha / \beta=2$ case over the other cases in Fig. 4 is quite apparent, cases that might be less than a third of the way in-between $\alpha / \beta=2$ and the two other would not be clearly superior, given the scatter of the points and the lack of shell-effect description in the theory. With this, we consider the locus of points in the $(\alpha, \alpha / \beta)$ plane corresponding the deviation reduced by a factor of $1 / 3$ compared to the optimal $\alpha / \beta=0$ point (i.e. $0.13 \mathrm{MeV}$ in Fig. 3), as the approximate boundary of the parameter range favored by the energy formula. Have we had a theory that could e.g. predict the shell effects, we might have been able to impose a more stringent limit on the parameters. For reference, we further show in Fig. 3 the boundary for the $0.40 \mathrm{MeV}$ deviation, passing through the optimal $\alpha / \beta=0$ point and in the vicinity of the optimal $\alpha / \beta=6$ point.

On one hand, the number of parameters here, in accessing the symmetry energy from the binding-energy data, represents a bare minimum, with the results necessarily being somewhat coarse. On the other hand, there can be advantages to the fits with a low parameternumber, besides the obvious immediate accessibility of the analysis. While coarse, the low parameter-number fits tend to be stable and faithful, without the optimization being thrown off by obscure features of data or by auxiliary theoretical inputs. As an example of dangers 
encountered when increasing the parameter number, when making the surface diffuseness $d$ in (29) a variable parameter [13], we find that the average deviation from measured energies minimizes for sensible parameter values. However, that minimum is found to be very flat when the diffuseness and symmetry parameters are varied simultaneously, extending far out into the region of large surface-to-volume parameter ratios and large diffuseness. Such behavior of a minimum marginalizes the significance of optimal values for a fit, given that, even when experimental errors are small, the theoretical uncertainties need to be incorporated [34]. The situation can be remedied by bringing in external knowledge [35], such as from electron scattering in the case in question. Such issues may be obvious for experimentalists, but are not routinely appreciated by theorists.

\section{ASYMMETRY SKINS}

\section{A. Interest in Skins}

Interest in asymmetry skins has surged in the context of the general interest in asymmetry effects, stemming from the availability of radioactive beams. Expectations were raised that an analysis of the asymmetry skins could permit to extrapolate the nuclear matter properties to the neutron matter [4-7]. Different features of effective interactions have been tested with regard to their bearing on the thickness of the skins. In several works [36], a direct connection between the neutron skin in the spherical ${ }^{208} \mathrm{~Pb}$ nucleus and the neutron star structure was made. Primarily, in recent times, the skins have been theoretically investigated within the microscopic structure calculations [37-39].

Unfortunately, experimental extraction of the neutron distributions has been difficult. Different methods have been employed, such as analyses of nucleon scattering [20,21,26,27] (including the analysis of polarized proton scattering and of neutron and proton scattering) or analyses combining information from strongly interacting $\left(p, \pi^{ \pm}, \alpha,{ }^{12} \mathrm{C}\right)$ and electromagnetic probes ( $e$ scattering, isotopic transition shifts) [22-25]. Recently, it has been proposed [40] to utilize parity violations in electron scattering to determine neutron rms radii, contributing to the surge of interest in the asymmetry skins.

\section{B. Skin Size}

To assess the thickness of the asymmetry skin for a given surface excess (25), let us consider the sharp sphere radii for neutrons $R_{n}$ and protons $R_{p}$. These radii correspond to volumes with interior densities such as for the bulk matter, i.e.

$$
N=\frac{N_{V}}{A} \rho_{0} \frac{4}{3} \pi R_{n}^{3}=N_{V}\left(\frac{R_{n}}{R}\right)^{3} \simeq N_{V}\left(1+\frac{3\left(R_{n}-R\right)}{R}\right) .
$$

In the last step, we use the fact that the intrinsic consistency of our considerations requires that we can tell the surface from the interior and, thus, must have $\left|R_{n}-R_{p}\right| \ll R$ or, equivalently, $\left|N_{S}-Z_{S}\right| \ll A$. We next get from (31)

$$
\frac{R_{n}-R}{R}=\frac{N_{S}}{3 N}
$$


With an analogous result for $R_{p}$, we find for the difference of the sharp-sphere radii:

$$
\frac{R_{n}-R_{p}}{R}=\frac{A\left(N_{S}-Z_{S}\right)}{6 N Z}=\frac{A}{6 N Z} \frac{N-Z-\frac{a_{C}}{12 \alpha} Z A^{2 / 3}}{1+\frac{\beta}{\alpha} A^{1 / 3}},
$$

where we inserted the result from (25). We see that the difference between the neutron and proton radii is primarily linear in the asymmetry and measures the symmetry coefficient ratio $\alpha / \beta$. For a surface with no capacitance for the asymmetry, characterized by $\alpha / \beta=0$, the asymmetry skin should vanish. If one were trying to compare the result (33) with the results in [14], one should note that there, in derivations, the approximation of $N \approx Z \approx A / 2$ is utilized.

Regarding (33), the results of measurements and of microscopic calculations for the skins are usually, however, not expressed in terms of the neutron-proton difference of sharp-sphere radii but rather the difference of rms radii. The difference between the rms compared to the sharp-sphere radii, aside from the standard reduction factor (dropping out from the ratio of radii) and from a correction for diffuseness, brings in a Coulomb correction additional to that already in (33). That additional correction stems from a polarization of the nuclear interior by the Coulomb forces. The polarization is schematically indicated in Fig. 2. In order to assess the polarization effect, within the net nuclear energy we represent the interior symmetry and Coulomb energies in integral forms. We expand these energies, respectively, to the second and first order in the deviation of asymmetry from uniformity:

$$
E=E^{\prime}+\frac{\alpha}{\rho_{0}} \int d \mathbf{r}\left(\delta \rho_{a}\right)^{2}-\frac{1}{2} \int d \mathbf{r} \Phi(r) \delta \rho_{a}
$$

Here, $\Phi$ is the Coulomb potential, $\rho_{a}$ is the neutron-proton density difference, $\rho_{a}=\rho_{n}-$ $\rho_{p}$, and we exploit the relation for the density changes $\delta \rho_{p}=-\delta \rho_{a} / 2$ following from the requirement $\rho_{n}+\rho_{p}=\rho_{0}$. The energy $E^{\prime}$ is the remaining portion of the net energy minimized with respect to the global partition of asymmetry between the interior and surface. The minimization of (34) with respect to $\delta \rho_{a}$, under the constraint of $\int d \mathbf{r} \delta \rho_{a}=0$, yields

$$
\delta \rho_{a}(r)=\frac{\rho_{0}}{4 \alpha}(\Phi(r)-\bar{\Phi})=\rho_{0} \frac{a_{C}}{8 \alpha} \frac{Z}{A^{1 / 3}}\left(1-\frac{5}{3} \frac{r^{2}}{R^{2}}\right),
$$

where the overline indicates an average over the interior volume and where we utilize the sharp-sphere potential $\Phi$. On calculating contribution of the deviation of asymmetry (35) from uniformity, to the difference of rms neutron and proton radii, we find

$$
\frac{\delta\left(\left\langle r^{2}\right\rangle_{n}^{1 / 2}-\left\langle r^{2}\right\rangle_{p}^{1 / 2}\right)}{\left\langle r^{2}\right\rangle^{1 / 2}}=-\frac{a_{C}}{168 \alpha} \frac{A^{5 / 3}}{N} .
$$

Notably, the contribution from the polarization (35) to the net energy is practically ignorable.

On combining (33) with (36) and on incorporating a correction for the surface diffuseness into (30), we get for the difference of the neutron and proton rms radii:

$$
\frac{\left\langle r^{2}\right\rangle_{n}^{1 / 2}-\left\langle r^{2}\right\rangle_{p}^{1 / 2}}{\left\langle r^{2}\right\rangle^{1 / 2}}=\frac{A}{6 N Z} \frac{N-Z}{1+\frac{\beta}{\alpha} A^{1 / 3}}-\frac{a_{C}}{168 \alpha} \frac{A^{5 / 3}}{N} \frac{\frac{10}{3}+\frac{\beta}{\alpha} A^{1 / 3}}{1+\frac{\beta}{\alpha} A^{1 / 3}}+\pi^{2} \frac{d\left(d_{n}-d_{p}\right)}{\left\langle r^{2}\right\rangle}
$$


Calculations [4] appear to indicate that, for lighter nuclei, the difference in the neutron and proton diffuseness $d_{n}-d_{p}$ is of the second order in the difference of sharp-sphere radii (33). For heavy nuclei, there may be a Coulomb contribution to the difference in the diffuseness. Overall, in any case, the correction for diffuseness is of a lower order in $A$ than the leading term in the skin thickness.

In Fig. 5, we compare the results of skin measurements in $\mathrm{Na}$ isotopes [25] to the results from (37) for different assumed values of $\alpha / \beta$, with the difference in diffuseness set to zero and with $\alpha$ made to follow the binding-energy correlation valley in Fig. 3. The data rather clearly rule out $\alpha / \beta<1$, but it is difficult, so far, to determine the energy parameter ratio with a much better precision. Further comparisons to data are shown in Figs. 6 and 7, for $\mathrm{Sn}$ and $\mathrm{Pb}$ isotopes, respectively. Overall, the displayed data appear to favor the ratios in the vicinity of $\alpha / \beta \sim 3$. As the charge number increases, one can see in Figs. 5-7, from the $\alpha / \beta=0$ results, the increasing importance of Coulomb corrections to the skin size. For realistic ratios $\alpha / \beta \lesssim 3$, we find that the Coulomb forces contribute about $0.07 \mathrm{fm}$ of reduction to the skin of ${ }^{208} \mathrm{~Pb}$. This can be compared to the $\sim 0.1 \mathrm{fm}$ change in the skin

size found by Furnstahl [7] for ${ }^{208} \mathrm{~Pb}$ when switching off the Coulomb interactions within mean-field models. The interior polarization and pushing out of the proton surface by the Coulomb interactions contribute about evenly to the skin reduction in our estimates.

It needs to be mentioned that, when different methods are employed in the skin extraction from measurements, such as in the case of Ca isotopes [20-23,27], the outcomes can easily differ by more than the claimed extraction errors, underscoring the difficulty in extractions. Results from a global fit of (37) to the available skin sizes obtained from data [20-27] are next shown in terms of contour lines (solid, slanted downward) in Fig. 3. As has been already discussed, the dependence of skin sizes on asymmetry primarily constrains the values of the ratio $\alpha / \beta$. We provide the contours for two combinations of a standard statistical deviation and an assumed average theoretical error $\delta$ of (37), i.e. $\chi^{2}=\chi_{\min }^{2}+1+\sum_{k}\left(\delta / \sigma_{k}\right)^{2}$ where $k$ is data index, and $\delta=0.02$ or $0.04 \mathrm{fm}$.

\section{Skin Size vs Difference of Separation Energies}

A strong dependence of the sizes of asymmetry skins on the proton-neutron difference of separation energies was predicted within the mean-field calculations of Ref. [41]. The dependence has been observed experimentally [25] and it was referred to in other mean-field calculations $[42,5]$. As we shall next demonstrate, this type of dependence naturally emerges in our results. The dependence can be used to constrain directly the surface parameter $\beta$.

The proton-neutron separation energy difference, up to a factor and $\mathcal{O}\left(A^{-1}\right)$ effects, is nothing else but the asymmetry chemical potential $\mu_{a}$. From the energy contributions (17) and (24), we get right away

$$
\mu_{a}=\frac{\partial E}{\partial\left(N_{S}-Z_{S}\right)}=\frac{2 \beta\left(N_{S}-Z_{S}\right)}{A^{2 / 3}}-\frac{5}{6} a_{C} \frac{Z}{A^{1 / 3}} .
$$

The difference of sharp-sphere radii (33) can be then written in terms of the chemical potential as

$$
\frac{R_{n}-R_{p}}{R}=\frac{A}{6 N Z}\left(N_{S}-Z_{S}\right)=\frac{\mu_{a}}{12 \beta} \frac{A^{5 / 3}}{N Z}+\frac{5}{72} \frac{a_{C}}{\beta} \frac{A^{4 / 3}}{N} .
$$


Turning to the difference of the r.m.s. radii, with the polarization contribution (36), the diffuseness correction and with $\mu_{a}=\left(S_{p}-S_{n}\right) / 2$, where $S_{p}$ and $S_{n}$ are the proton and neutron separation energies, we find

$$
\frac{\left\langle r^{2}\right\rangle_{n}^{1 / 2}-\left\langle r^{2}\right\rangle_{p}^{1 / 2}}{\left\langle r^{2}\right\rangle^{1 / 2}}=\frac{S_{p}-S_{n}}{24 \beta} \frac{A^{5 / 3}}{N Z}-\frac{a_{C}}{168 \alpha} \frac{A^{5 / 3}}{N}\left(1-\frac{35}{3} \frac{\alpha}{\beta A^{1 / 3}}\right)+\pi^{2} \frac{d\left(d_{n}-d_{p}\right)}{\left\langle r^{2}\right\rangle} .
$$

If not for the Coulomb correction, the surface asymmetry in (38) would have been proportional to the difference of separation energies, with the coefficient of proportionality inversely proportional to $\beta$. The linearity with respect to the separation-energy difference is largely retained for the skin size in (40). (Principally, one might strive in Eq. (40) to eliminate the separate dependence on $N$ and $Z$, in favor of exclusively $S_{n}-S_{p}$ and $A$, but the separate dependence on $N$ and $Z$ appears only in correction terms, either associated with the Coulomb forces or geometry, so it is not crucial.) Into the results previously compared to data, i.e. the dependence of energy and of skin size on mass and charge numbers, the surface and volume symmetry coefficients entered in a combination. By contrast, the dependence of skin size on the separation energy difference, that we now examine, tests primarily the surface symmetry coefficient. The difference between the cases is easily understood following the capacitor analogy. Thus, the volume and surface coefficients characterize the surface and volume capacitances, respectively. Either the net energy or the ratio of the surface-to-net asymmetry depended on the net capacitance. However, in the capacitor analogy, the separation-energy difference represents a voltage and the relation between surface charge and the voltage depends only on the surface capacitance (as a matter of capacitance definition).

We now confront the result (40) with the data on skin sizes as a function of separationenergy difference for $\mathrm{Na}, \mathrm{Sn}$ and $\mathrm{Pb}$ nuclei in Figs. 8, 9 and 10, respectively. The data appear to favor the values $\beta \gtrsim 10 \mathrm{MeV}$. In fitting all data on skin sizes [20-27], now with Eq. (40), we again generate contour lines (solid, slanted upward) in the plane of $\alpha-\alpha / \beta$ in Fig. 3, for $\chi^{2}=\chi_{\min }^{2}+1+\sum_{k}\left(\delta / \sigma_{k}\right)^{2}$ where $k$ is data index, and the systematic error equal either to $\delta=0.02$ or $0.04 \mathrm{fm}$. Since the dependence primarily constraints $\beta$, the constraint lines in the plane $\alpha-\alpha / \beta$ are approximately straight lines passing through the origin of the coordinate set.

\section{Symmetry Parameter Values}

With the three dependencies fitted to the data, we now have three regions in the plane of Fig. 3, favored by the data, that intersect. Since the separation energies stem from the binding energies, one would expect that the intersection of the two regions derived from skin sizes would be well centered over the region favored by binding energies. Some offset may be from a bias due to the fact that the skin data primarily stem from nuclei near closed shells or due to the the fact that the surface diffuseness is ignored in the Coulomb corrections for skin size but is not ignored in the binding-energy formula. In any case, the offset suggests that we may need to use bias errors as large as $\delta=0.03 \mathrm{fm}$ for Eqs. (37) and (40). Intersection of the three regions favored by data then yields the following limits on the parameter values: $2.0 \lesssim \alpha / \beta \lesssim 2.8$ for the ratio, $27 \mathrm{MeV} \lesssim \alpha \lesssim 31 \mathrm{MeV}$ for the volume 
and $11 \mathrm{MeV} \lesssim \beta \lesssim 14 \mathrm{MeV}$ for the surface. Within those limits, though, the parameters are correlated, cf. Fig. 3.

It should be mentioned that values of the volume symmetry parameters $\alpha$ in some advanced mass descriptions fall within our region, such as in [43] with $J=28 \mathrm{MeV}$ and in [13] with their $-\kappa_{v o l} b_{v o l}=29.3 \mathrm{MeV}$. However, in [43] the particular parameter value is set and in neither of those sources the uncertainties are actually assessed. Elsewhere, values outside of our range are followed [12] and in the mean-field structure calculations, based on Skyrme interactions or Lagrangians, a wide range of the symmetry volume parameters is employed [7], from 24.5 to $43.5 \mathrm{MeV}$. Wide parameter ranges are also employed in various phenomenological considerations such as for the nuclear liquid-gas phase-transition [44]. On the other hand, nuclear-matter calculations relying on realistic nucleon-nucleon interactions appear to yield volume symmetry energy values within our range, of 27-29 MeV depending on the interaction in [45] and $30.10 \mathrm{MeV}$ in [46]. Those microscopic energies, however, are obtained as the difference between the neutron and symmetric nuclear-matter energies but this, normally, is an excellent approximation to the symmetry energy from $N-Z$ expansion.

Regarding the obtained values of the ratio of $\alpha / \beta$, one may ask about a justification for the large- $A$ expansion of the symmetry energy in mass formulas $[12,13]$. With the expansion amounting to an approximation in $(28)$ of $1 /(1+x)=1-x+x^{2}-\ldots \approx 1-x$, where $x=\alpha /\left(\beta A^{1 / 3}\right)$, we may adopt a criterion that the expansion is justified when the correction to the effect of surface on symmetry energy, from ignoring higher-order terms, is less than 20\%. This requires $x<0.2$ or $A>(5 \alpha / \beta)^{3} \geq 1000$ which is never satisfied. (Even when the next term in the expansion is included [13], meeting the criterion necessitates $A>5^{3 / 2}(\alpha / \beta)^{3}=(90-250)$, depending on the $\alpha / \beta$ ratio.) In the droplet model fit [12] to binding energies and fission barriers, with nonexpanded symmetry energy, the value of the ratio $\alpha / \beta$ is within our region, as represented by $\frac{9}{4} \frac{J}{Q}=2.5$, and so is the value of

the surface parameter $\beta$, represented by $\frac{4}{9} Q=13.0 \mathrm{MeV}$. However, the uncertainties are not produced there and, moreover, with an inclusion of the volume symmetry parameter, $J=32.7 \mathrm{MeV}$, the results are well outside of our correlation region. In earlier mass and barrier fits within the droplet model, the ratio itself and the surface parameter were much different, with $\frac{9}{4} \frac{J}{Q}=4.9$ and $\frac{4}{9} Q=7.5 \mathrm{MeV}$ in [16], respectively. It would be interesting to find out the value of the surface symmetry coefficient for the advanced mass calculations in [43]. In [13], the volume-to-surface symmetry parameter ratios $\alpha / \beta$ for mass fits are $-b_{\text {surf }} \kappa_{\text {surf }} /\left(b_{\text {vol }} \kappa_{v o l}\right)=1.31$ and 1.35 , but these results are for an expanded form of the symmetry energy, at two different levels of the expansion.

In the next section, we employ our elementary results in the description of asymmetry oscillations in nuclei.

\section{APPLICATION TO ASYMMETRY OSCILLATIONS}

\section{A. Goldhaber-Teller and Steinwedel-Jensen Models of the Giant Dipole Resonance}

So far, we discussed elementary static properties of ground-state nuclei. Nuclear excitations may involve displacements of protons relative to neutrons, compared to the ground state, with prominent giant resonance structures in cross sections allowing for an interpretation in terms of a collective motion involving a number of nucleons. The lowest in energy (or 
frequency) is the giant dipole resonance (GDR) prominent in the low-energy photoabsorption [47] and inelastic electron-scattering [48] cross-sections.

Two early models have been advanced for the GDR, under the names of Goldhaber and Teller (GT) [29] and Steinwedel and Jensen (SJ) [29,30]. In the GT model, the neutron and proton distributions are assumed to oscillate against each other as rigid entities, see Fig. 11. Since the potential energy associated with the displacement is proportional to the nuclear surface area, i.e. $\propto A^{2 / 3}$, while the moving mass is proportional to $A$, the resonance excitation energy in this model is proportional to $A^{-1 / 6}: E_{G D R}=\hbar \Omega \propto \sqrt{A^{2 / 3} / A}=A^{-1 / 6}$. In the SJ model, a standing wave of neutron vs proton displacement develops within the nuclear volume, satisfying the surface condition of vanishing asymmetry-flux, analogous to the closed-pipe condition in the standard example of oscillations for a continuous medium. As the wavelength is then in a definite constant ratio to the nuclear radius, the resonance energy in the model is proportional to $A^{-1 / 3}: E_{G D R}=\hbar c_{a} / \lambda \propto A^{-1 / 3}$, where $c_{a}$ is the speed of propagation for asymmetry perturbations in normal symmetric matter.

In detail, within the SJ model [30,49], it is assumed that there is no net movement of matter, i.e. the net matter velocity $\mathbf{v}$ vanishes and the net nucleon density stays intact, consistent with the continuity equation: $\partial \rho / \partial t=-\nabla(\rho \mathbf{v})$. The relative density $\rho_{a}=\rho_{n}-\rho_{p}$ changes, but the state of matter is locally the same as within a ground-state nucleus, just at the new asymmetry. The vanishing net velocity implies a relation between the velocities for individual particles, $\mathbf{v}_{n}$ and $\mathbf{v}_{p}$, and densities, following from the consideration of fluxes that need to meet the condition

$$
0=\rho \mathbf{v}=\rho_{n} \mathbf{v}_{n}+\rho_{p} \mathbf{v}_{p}
$$

This then implies that the flux in the asymmetry continuity equation may be expressed in terms of the relative velocity $\mathbf{v}_{n p}=\mathbf{v}_{n}-\mathbf{v}_{p}$,

$$
\frac{\partial \rho_{a}}{\partial t}=-\nabla\left(\rho_{n} \mathbf{v}_{n}-\rho_{p} \mathbf{v}_{p}\right)=-\nabla\left(\frac{2 \rho_{n} \rho_{p}}{\rho} \mathbf{v}_{n p}\right) \simeq-\frac{2 N Z \rho_{0}}{A^{2}} \nabla \mathbf{v}_{n p} .
$$

where the approximate equality is valid in the lowest order in the disturbance. An Euler type equation for the relative velocity can be next used to close the equation above,

$$
\frac{\partial}{\partial t} \mathbf{v}_{n p}=-\frac{2}{m^{*}} \nabla \mu_{a}=-\frac{2}{m^{*}} \frac{\partial \mu_{a}}{\partial \rho_{a}} \nabla \rho_{a}=-\frac{2 c_{a}^{2}}{\rho_{0}} \nabla \rho_{a}
$$

where $m^{*}$ is the effective mass and

$$
c_{a}^{2}=\frac{\rho_{0}}{m^{*}} \frac{\partial \mu_{a}}{\partial \rho_{a}} .
$$

On differentiating Eq. (42) with respect to time and on substituting (43), we get the wave equation for the asymmetry density

$$
\frac{\partial^{2}}{\partial t^{2}} \rho_{a}=\frac{4 N Z}{A^{2}} c_{a}^{2} \nabla^{2} \rho_{a}
$$

Under spherical symmetry, the normal vibrations are looked for, in the standard way, in terms of the deviation from ground-state density expressed as the product of a spherical Bessel function $j_{\ell}$ and of a spherical harmonic $Y_{\ell m}$, 


$$
\delta \rho_{a}=D_{V} \sin (\omega t) j_{\ell}(q r) Y_{\ell m}(\Omega)
$$

where, following (45), the wavenumber $q$ and the frequency $\omega$ are related by

$$
\omega=\frac{2 \sqrt{N Z}}{A} c_{a} q
$$

The radial velocity component, from (43) and (46), is

$$
v_{n p}^{r}=\frac{2 D_{V}}{\rho_{0}} \frac{A}{2 \sqrt{N Z}} c_{a} \cos (\omega t) j_{\ell}^{\prime}(q r) Y_{\ell m}(\Omega)
$$

The condition that the normal component of flux vanishes at the surface [28], $v_{n p}^{r}(R, \Omega)=0$, yields the wavenumber values

$$
q_{\ell n}=\frac{j_{\ell n}^{\prime}}{R}
$$

where $n$ orders the values and $j_{\ell n}^{\prime}$ represents the $n$ 's zero of the spherical Bessel function derivative, $j_{\ell}^{\prime}\left(j_{\ell n}^{\prime}\right)=0$. For the resonance energy, we get, given (47),

$$
E_{\ell n}=\hbar \omega_{\ell n}=\frac{2 \sqrt{N Z}}{A} \hbar c_{a} q_{\ell n}=\frac{2(N Z)^{1 / 2}}{A^{4 / 3}} \frac{\hbar c_{a} j_{\ell n}^{\prime}}{r_{0}}
$$

where, with (44) and (16),

$$
c_{a}=\sqrt{\frac{2 \alpha}{m^{*}}} .
$$

The GDR is characterized by $\ell=1$ and $n=1$. With $j_{11}^{\prime} \simeq 2.08$, the GDR energy in the SJ model is then, finally,

$$
E_{G D R}=\frac{2(N Z)^{1 / 2}}{A^{4 / 3}}\left(\frac{2 \alpha}{m^{*}}\right)^{1 / 2} \frac{2.08 \hbar}{r_{0}} \approx\left(\frac{2 \alpha}{m^{*}}\right)^{1 / 2} \frac{2.08 \hbar}{r_{0} A^{1 / 3}} .
$$

Within the GT model of the GDR, the changes in asymmetry occur only within the nuclear surface and in the SJ model only within the interior. Experimental dependence of GDR energy on mass number, displayed in Fig. 12, is intermediate [47] between that characteristic for the GT and SJ models, suggesting that both the volume and surface participate in the oscillations. To explain the mass dependence of the GDR energy, in Ref. [50] the oscillation in the SJ model was treated as an elementary mode of nuclear oscillation coupled to a second GT mode of oscillation, within the same nucleus. As the resonance frequencies for the two models, GT and SJ, shifted with a changing nuclear mass, the lower-frequency normal mode for the combination of oscillators changed from a mode with a predominantly GT to SJ content. However, this casts the nuclear system into one with only two degrees of freedom. The physical situation is rather that of a continuous system for which, though, the surface can generally accept the asymmetry flux, making the original SJ boundary condition excessively restrictive. 


\section{B. Description of Asymmetry Oscillations}

We now explore the modification and consequences of the surface boundary condition for the asymmetry oscillations. Given that the surface can store an asymmetry excess, we replace the SJ condition of a vanishing flux with the condition for the change in surface asymmetry density $\mathrm{n}_{a}$,

$$
\frac{\partial}{\partial t} \mathrm{n}_{a}=\rho_{n} v_{n}^{r}-\rho_{p} v_{p}^{r}=\frac{2 N Z \rho_{0}}{A^{2}} v_{n p}^{r}
$$

cf. (42). As the chemical potential for the surface needs to be the same as within the adjacent interior, this can be further rewritten as a condition involving the interior chemical potential, or involving the volume density next to the surface,

$$
\frac{2 N Z \rho_{0}}{A^{2}} v_{n p}^{r}=\frac{\partial}{\partial t} \mathrm{n}_{a}=\frac{\partial \mathrm{n}_{a}}{\partial \mu_{a}} \frac{\partial \mu_{a}}{\partial t}=\frac{\partial \mathrm{n}_{a}}{\partial \mu_{a}} \frac{\partial \mu_{a}}{\partial \rho_{a}} \frac{\partial \rho_{a}}{\partial t} .
$$

When the surface does not accept the excess, i.e. $\partial \mathrm{n}_{a} / \partial \mu_{a}=0$, we get the regular SJ condition of a vanishing flux.

Looking for normal modes with (46) and

$$
\delta \mathrm{n}_{a}=D_{S} \sin (\omega t) Y_{\ell m}(\Omega)
$$

we find from the requirement of equality for chemical potentials at the surface, in accordance with local surface and volume densities of symmetry energy, that

$$
D_{S}=\frac{\alpha}{3 \beta A^{1 / 3}} D_{V} R j_{\ell}(q R)
$$

Moreover, with (48) and (47), we can obtain from either (53) or (54) the condition for the wavevector of oscillation:

$$
q R j_{\ell}(q R)=\frac{3 \beta A^{1 / 3}}{\alpha} j_{\ell}^{\prime}(q R)
$$

As the ratio $\alpha / \beta A^{1 / 3}$ grows from small to large values, the relative magnitude of the surface amplitude $D_{S}$ in (56) grows. Moreover, it is apparent that the solutions to the wavenumber equation (57) generally shift then from $j_{\ell n}^{\prime} / R$ to $j_{\ell n} / R$, where $j_{\ell n}$ represents the $n$ 'th zero of the spherical Bessel function, $j_{\ell}\left(j_{\ell n}\right)=0$. In terms of the standard example of oscillations for a continuous medium, the boundary condition shifts from one representing a closed pipe to one representing an open pipe. Coping with the $n=1, \ell>0$ solution to the wavenumber equation at $q \rightarrow 0$, as $\beta A^{1 / 3} / \alpha \rightarrow 0$, however, requires a careful expansion.

We concentrate next on the $\ell=1$ GDR case. For $\alpha / \beta A^{1 / 3} \rightarrow 0$, the surface amplitude in (56) tends to zero and we arrive at the SJ limit with the GDR energy given by (52). For $\beta A^{1 / 3} / \alpha \rightarrow 0$, we need to consider the Bessel function in (57) at small values of its argument where $j_{1}(x) \approx(x / 3)\left(1-x^{2} / 10\right)$. We then find for the wavenumber

$$
q_{11} \simeq\left(\frac{3 \beta}{\alpha}\right)^{1 / 2} \frac{1}{r_{0} A^{1 / 6}}
$$


and for the resonance energy

$$
E_{G D R}=\hbar \omega_{11}=\frac{2 \sqrt{N Z}}{A} \hbar c_{a} q_{11}=\frac{2(N Z)^{1 / 2}}{A^{7 / 6}}\left(\frac{6 \beta}{m^{*}}\right)^{1 / 2} \frac{\hbar}{r_{0}} \approx\left(\frac{6 \beta}{m^{*}}\right)^{1 / 2} \frac{\hbar}{r_{0} A^{1 / 6}} .
$$

In this limit, the resonance energy becomes dependent only on the surface symmetry parameter $\beta$ and not on the volume parameter $\alpha$. Moreover, the volume amplitude gets suppressed relative to the surface amplitude in (56). This is clearly the GT limit of vibration.

Figure 12 shows next fits to the data [47] on mass dependence of the GDR energy, with $c_{a}$ treated as a fit parameter and with $q_{11}$ obtained from (57) for different values of $\alpha / \beta$. We see that a realistic ratio of $\alpha / \beta=2.5$ yields a much better description of the data than the SJ ratio of $\alpha / \beta=0$. However, a still larger ratio than 2.5 would provide an even better description. It is possible that other factors than the finite capacitance of the surface for asymmetry play a role here, such as the local deviations of a system from the ground state [51]. For the best fit with $\alpha / \beta=2.5$, we get $c_{a}=0.28 c$, corresponding to $m=0.84 \mathrm{~m}^{*}$.

One may ask for what values of $A$ and realistic $\alpha / \beta$ the vibrations turn from more GT to more SJ like. As a criterion, one may use the fraction of potential energy of the vibration attributable to the surface. With this criterion, one finds that the transition takes place at $\beta A_{t r}^{1 / 3} / \alpha=3 / 2$, and, thus, for the range of $2.0 \lesssim \alpha / \beta \lesssim 2.8$, at $30 \lesssim A_{t r} \lesssim 75$. The relatively high transition masses can be attributed to the node of the vibration at the center of a nucleus.

\section{Transition Densities}

The profile of a standing wave in an oscillation represents, at the quantal level, the transition density for the resonance. This density is probed in inelastic electron scattering [48]. To our knowledge, no inversion of data for the GDR transition density has been ever made, but the data were tested against the GT and SJ type densities, with a conclusion that the GDR (and also $\ell>1$ ) densities are in-between the two limits [48]. Even in theoretical works, the transition densities have not been much quoted.

We write the transition density as

$$
\rho_{1}(\mathbf{r})=\rho_{1}(r) Y_{\ell m}
$$

where $\rho_{1}(r)$ includes both the volume and surface contributions. Interpreting the volume results in terms of long-wavelength modulations of the particle densities and the surface results in terms of the modulations of radii, we have for the density [48]

$$
\rho_{1}(r)=\frac{1}{\rho_{0}}\left[D_{V} j_{\ell}(q r) \rho(r)-D_{S} \frac{d \rho}{d r}\right]
$$

where $\rho(r)$ is the ground-state density distribution. With (56), we can further rewrite this as

$$
\rho_{1}(r)=\frac{D_{V}}{\rho_{0}} j_{\ell}(q r)\left[\rho(r)-\frac{\alpha}{3 \beta A^{1 / 3}} r \frac{d \rho}{d r}\right]
$$


Establishing a contact with microscopic theory, for ${ }^{40} \mathrm{Ca}$ in Fig. 13, we compare our GDR transition density (62), with (30), to the transition density from the microscopic calculations of Ref. [52], that include the effects of $2 \mathrm{p}-2 \mathrm{~h}$ excitations and of ground-state correlations. The normalization in (62) is obtained from the Thomas-Reiche-Kuhn sum rule; for illustrative purposes, we take $m^{*}=m$ as in [52]. The calculations [52] employ a phenomenological Landau-Migdal type interaction in use in describing different giant resonances. We note that a best contact with our results is made for $\alpha / \beta \gtrsim 2.5$.

\section{DENSITY DEPENDENCE OF SYMMETRY ENERGY}

Several works tried to identify links between the asymmetry skins and bulk asymmetry properties of nuclear matter, within microscopic theory [4-7]. Having explored a link between the skins and the symmetry parameters, we will now try to establish a connection between the parameters and the density dependence of symmetry energy, starting with the ThomasFermi theory.

\section{A. Symmetry Parameters in the Thomas-Fermi Theory}

We shall consider a nuclear energy functional expanded to the second order in asymmetry:

$$
E=E_{0}+\int d \mathbf{r} \rho S(\rho)\left(\frac{\rho_{a}}{\rho}\right)^{2} \equiv E_{0}+E_{a} .
$$

Here we assume that the expansion term in asymmetry is local, but this will not be essential for much of the discussion that follows. The function $S$ is the density-dependent symmetry energy per nucleon and $S\left(\rho_{0}\right)=\alpha$. The energy $E_{0}$ is generally nonlocal but independent of asymmetry as the zeroth-order term in the expansion; since the Coulomb effects have been explicitly considered in Secs. II and III, we will disregard them for the discussion that follows. Regarding the role of asymmetry in any nonlocality, there had been indications $[4,7,53]$ that it has a negligible impact on the properties of nuclei; the apparent success in systematizing the asymmetry skins in terms of the nuclear-matter properties [4-7] lends, in itself, a support to the weak impact.

A simple example of the functional (63) is such as employed in the initializations of transport calculations for central reactions [54]:

$$
\begin{aligned}
E_{0} & =\int d \mathbf{r}\left(\frac{3}{5} \epsilon_{F} \rho+\frac{\mathcal{A} \rho^{2}}{\rho_{0}}+\frac{\mathcal{B} \rho^{\sigma+1}}{(\sigma+1) \rho_{0}^{\sigma}}\right)+\frac{\mathcal{D}}{2 \rho_{0}} \int d \mathbf{r}(\nabla \rho)^{2} \\
S & =\frac{1}{3} \epsilon_{F}+\frac{\mathcal{C} \rho}{\rho_{0}}+\frac{\mathcal{F} \rho^{2}}{\rho_{0}^{2}}
\end{aligned}
$$

with the Fermi energy $\epsilon_{F}=\left(3 \pi^{2} / 2\right)^{2 / 3} \hbar^{2} \rho^{2 / 3} / 2 m$. The constants $\mathcal{A}, \mathcal{B}$ and $\sigma$ are fixed by the requirement that the functional yields the energy minimum of $E / A=a_{V} \approx-16 \mathrm{MeV}$ at $\rho_{0}$ in symmetric nuclear matter, characterized by a specific incompressibility constant $K$. E.g. for $K=260 \mathrm{MeV}$, we get $\mathcal{A}=-180.9 \mathrm{MeV}, \mathcal{B}=128.1 \mathrm{MeV}$ and $\sigma=1.446$. The constant $\mathcal{D}$ provides a size scale for the surface diffuseness and an adequate surface profile 
is obtained [54] for $\mathcal{D} \approx 20.4 \mathrm{MeV} \mathrm{fm}^{2}$. As to the constants in $S$, we have the relation $\alpha=\epsilon_{F}\left(\rho_{0}\right) / 3+\mathcal{C}+\mathcal{F}$ and, otherwise, different combinations of the constants $\mathcal{C}$ and $\mathcal{D}$ yield different values of $\beta$ as will be discussed.

The minimization of the energy (63) under the conditions of fixed particle numbers

$$
A=\int d \mathbf{r} \rho \quad \text { and } \quad N-Z=\int d \mathbf{r} \rho_{a}
$$

produces the Thomas-Fermi equations:

$$
\begin{aligned}
\mu & =\frac{\delta E_{0}}{\delta \rho}+\rho_{a}^{2} \frac{d}{d \rho} \frac{S}{\rho}, \\
\mu_{a} & =2 \rho_{a} \frac{S(\rho)}{\rho} .
\end{aligned}
$$

At first, those equations will just serve as a background illustration for the claims made in the discussion.

On the basis of (63), we would like to find out changes in the nuclear ground-state energy, as the particle asymmetry changes. First, we consider a symmetric system and find $\rho(\mathbf{r})$ that minimizes the energy $E=E_{0}$. With the energy $E$ being quadratic in asymmetry, the net density minimizing the energy functional at a finite asymmetry will differ from the density for the symmetric system only to within a second order in asymmetry. With this, to within the second order in asymmetry, the ground-state $E_{a}$ in (63) may be calculated with $\rho(\mathbf{r})$ obtained for symmetric matter. Moreover, since the symmetric-system $\rho(\mathbf{r})$ minimizes $E_{0}$, any changes to it, of the second order in asymmetry, will produce only quartic terms of change in $E_{0}$. In conclusion, if we are interested in the change in the ground-state energy to the second order in asymmetry, we just need to consider the $E_{a}$ term in (63) and we can use there the density $\rho(\mathbf{r})$ from the energy $E_{0}$ minimized for a symmetric system. In terms of the Thomas-Fermi equations, this means that we can uncouple (67) by putting $\rho_{a}=0$ there.

From the second of the Thomas-Fermi equations, we have in the ground-state system

$$
\rho_{a}=\frac{\mu_{a}}{2} \frac{\rho}{S}
$$

and from (63)

$$
E_{a}=\frac{\mu_{a}^{2}}{4} \int d \mathbf{r} \frac{\rho}{S}
$$

Within the analogy with a capacitor, the integral $\int d \mathbf{r}(\rho / 2 S)$ represents the system capacitance, independent of the deposited charge. The volume capacitance is $A / 2 \alpha$ and the reminder is the surface capacitance:

$$
\frac{A^{2 / 3}}{2 \beta} \equiv \frac{\mathcal{S}}{2 \pi r_{0}^{2} \beta}=\frac{1}{2} \int d \mathbf{r} \rho\left(\frac{1}{S}-\frac{1}{\alpha}\right) .
$$

As we will not be pursuing here curvature effects, we just turn to the surface capacitance for a semiinfinite matter. With $x$ a coordinate along the direction perpendicular to the surface 
and on factoring out the density dependence from the symmetry energy with $S=\alpha s\left(\rho / \rho_{0}\right)$, we find from (71)

$$
\frac{\alpha}{\beta}=\frac{3}{r_{0}} \int d x \frac{\rho}{\rho_{0}}\left(\frac{1}{s}-1\right) .
$$

Thus, for (63), given that the density profile for the surface is constrained, the ratio of the symmetry parameters $\alpha / \beta$ is a measure for the density dependence of the symmetry energy $S$. We note that a density-independent symmetry energy, characterized by $s \equiv 1$, yields $\alpha / \beta=0$. The falling density at the surface produces in this case no extra capacitance per nucleon compared to the interior. The faster the drop of $s$ with density, the larger the extra capacitance, exhibited in a larger $\alpha / \beta$ ratio.

We next test the validity of the considerations from Secs. II and III within a Thomas Fermi model based on Eqs. (64) and (65), with a minor alteration, however, that the different neutron and proton Fermi energies are treated explicitly for asymmetric nuclei. On one hand, we solve the differential equation [54] from (67) for the semiinfinite symmetric matter and we vary $\mathcal{C}$ and $\mathcal{F}$ to get various combinations of $\alpha$ and $\alpha / \beta$ from (72), requiring that they follow the binding-energy correlation from Fig. 3. Given the $\alpha$ and $\beta$ values, we make a prediction for the size of the asymmetry skin of a specific nucleus, following Eq. (37) with $d_{n}-d_{p}=0$. On the other hand, we solve the Thomas-Fermi equations directly for a nucleus, with Coulomb forces included, and calculate the difference of rms radii. A comparison of the results for $\mathrm{Na}$ isotopes is shown in Fig. 14. The results are both compared when changing the isotope mass, while keeping the symmetry parameters fixed, and for one isotope when changing the symmetry parameters. As is seen, the agreement of the estimate (37) with the actual skin size is rather remarkable. For heavy nuclei, however, a small systematic difference develops between the actual and estimated skin sizes, that largely vanishes when the Coulomb forces are switched off. The size of the discrepancy, $\sim 0.03 \mathrm{fm}$ for $\mathrm{Pb}$, for which we have no understanding, is compatible with the possible difference between our estimate of the Coulomb effects in Eq. (37) and that of Furnstahl [7] (already brought up in Sec. III).

A very recent, more involved, analysis of surface symmetry energy and skin sizes, within the Thomas-Fermi theory, has been carried out by Bodmer and Usmani in Ref. [53], with some conclusions comparable to those that emerge here. In detailed comparisons of their results to ours, it should be noted that their surface symmetry energy and parameter are differently defined than ours for a finite nucleus and e.g. include Coulomb contributions.

\section{B. Constraints on Density Dependence of Symmetry Energy}

To gain a perspective on the recent microscopic results on skin systematics in the literature [5-7], we further confront our results from the Thomas-Fermi model, to those obtained in the variety of mean-field models explored there. In Fig. 15, the skins from the mean-field calculations for ${ }^{208} \mathrm{~Pb}$ [7] (symbols) are presented vs the reduction factor $s$ for the symmetry energy per nucleon at $\rho_{0} / 2$ (left panel), vs the scaled derivative of the energy at normal density $p_{0}=\rho_{0}^{2} d S /\left.d \rho_{0}\right|_{\rho_{0}}$ (center panel) and vs the volume symmetry parameter $\alpha$ (right panel). The skin dependence on $p_{0}$ and on $\alpha$ was explored in [7]. A similar derivative to

$p_{0}$ was utilized in $[5,6]$. Moreover in Fig. 15, the results from the Thomas-Fermi model 
are presented (lines) for the symmetry parameters changing along the correlation valley in Fig. 4. It is apparent in each panel of Fig. 15, that the Thomas-Fermi model produces general trends such as found in the mean-field calculations. However, in the three panels, the represented dependencies for skins in the Thomas-Fermi model are of a quite different nature. Thus, when $\alpha$ just provides a normalization for the symmetry energy, it has a very limited bearing on the skins in (37), merely through a rescaling of the Coulomb corrections (see also [53]). However, when $\alpha$ is correlated with $\alpha / \beta$ through the binding-energy requirements, the changes in $\alpha$ force changes in $\alpha / \beta$ and, thus, produce significant changes in the skin in the rightmost panel of Fig. 15. In the center panel for the Thomas-Fermi model, we observe combined indirect and direct (albeit at the edge of the relevant density interval) effects of the abscissa on the skin. Only in the leftmost panel for the Thomas-Fermi model, we observe the effect of a direct physical connection between the abscissa and the skin, through Eqs. (37) and (72). The three panels illustrate potential dangers of scouting for a physical relation [5-7] looking at numerical results within models that are necessarily constricted.

For a given effective interaction, the mean-field models likely yield more realistic results in the surface region than the Thomas-Fermi model. However, there is no reason to believe that a strong primary sensitivity to the density dependence of $s$ disappears, for the symmetry parameter ratio $\alpha / \beta$, in the mean-field models. Using the results [7] for the ${ }^{208} \mathrm{~Pb}$ skins in those models, we can assess $\alpha / \beta$ for the models, from (37), and we can produce constraints on the low-density $s$ from the constraints on $\alpha / \beta$. An alternative would be to use the data on ${ }^{208} \mathrm{~Pb}$ skins directly to constrain $s$ in Fig. 15. In Fig. 16, the values of $s$ at $\rho_{0} / 2$ are shown, for the mean-field models, vs $\alpha / \beta$ from inverting (37). In the inversion, basing here the results on one heavy nucleus, we augment the Coulomb correction in (37) by $0.03 \mathrm{fm}$; an ad hoc procedure suitable for all nuclei might be to amplify the Coulomb correction by a factor of 1.4. Given the variation of the results in Fig. 16, for the acceptable range of $2.0 \lesssim \alpha / \beta \lesssim 2.8$, we deduce the range of the symmetry-energy reduction factor at $\rho_{0} / 2$ of $0.57 \lesssim s \lesssim 0.83$. Compared to the previous unbiased limit [7] on $p_{0}$, of $1 \mathrm{MeV} / \mathrm{fm}^{3}<p_{0}<$ $4 \mathrm{MeV} / \mathrm{fm}^{3}$, our limit on $(1-s)$ represents a nearly twofold improvement in constraining the low-density dependence of symmetry energy. The $s$-shift is for the fairness in comparison; note that the majority of theoretical calculations in Fig. 16 suggests an even narrower range for $s$ than above, of $0.67 \lesssim s \lesssim 0.83$. Concerning the microscopic nuclear-matter calculations with realistic nucleon-nucleon interactions, minimally extrapolating underneath the density range in [45], the reduction in symmetry energy at $\rho_{0} / 2$, for the variety of interactions there, is within our narrow reduction range, at 0.67-0.73. However, in the calculations of Ref. [46], the reduction in symmetry energy at $\rho_{0} / 2$ is only within our wider range, at 0.59 .

\section{CONCLUSIONS}

Binding in the symmetric nuclear matter can be realistically assessed by anyone with a minimal experience, using the textbook mass formula and a sample of nuclear masses. We hope to have demonstrated that the information on symmetry energy can be accessed with a nearly comparable ease, using a minimally modified formula. Elementary but general considerations, for the nucleus as a macroscopic system, indicate that the dependence of surface tension on nuclear asymmetry necessarily implies the emergence of an asymmetry skin. In the lowest order in asymmetry, the surface tension and surface energy need to be 
quadratic in the asymmetry. With analogous behavior of the volume energy, the surface and volume symmetry energies combine as energies of two connected capacitors with the volume capacitance proportional to the volume and the surface capacitance proportional to the area.

In minimizing the net energy, the net asymmetry partitions itself into the surface and volume asymmetry in proportion to the capacitances. As nuclear size increases, the surface portion of the asymmetry decreases, as surface decreases relative to the volume. The minimal net symmetry energy is given in terms of the net asymmetry squared divided by the sum of the volume and surface capacitances.

In analyzing the binding energies, we demonstrate that the surface symmetry contribution is required to describe the energies of light asymmetric nuclei at a level similar to that typical for other nuclei. Overall, however, the binding energies are better in constraining a combination of the coefficients for the surface and volume symmetry energies, than in constraining the individual coefficients, at least at the level of our simple formula. The specific coefficient combination represents the symmetry coefficient in the standard mass formula. On the other hand, the dependencies of skin sizes on asymmetry and on the neutron-proton separation-energy difference primarily constrain, respectively, the ratio of the symmetry volume to surface parameters and the surface symmetry parameter. Using the three types of constraints, we establish limits on the volume symmetry parameter of $27 \mathrm{MeV} \lesssim \alpha \lesssim 31 \mathrm{MeV}$, on the surface symmetry parameter of $11 \mathrm{MeV} \lesssim \beta \lesssim 14 \mathrm{MeV}$, and on the parameter ratio, of $2.0 \lesssim \alpha / \beta \lesssim 2.8$. Within those limits, however, the parameters are correlated. In analyzing the skin sizes, we provide straightforward interpretations for regularities observed both in measurements and in microscopic structure calculations.

For reference, when taking the parameter ratio in the middle of the permitted range, at $\alpha / \beta \simeq 2.4$, the sample best-fit parameter values in describing the binding energies with (28) and (29) are $a_{V}=15.4808 \mathrm{MeV}, a_{S}=18.4359 \mathrm{MeV}, a_{C}=0.696424 \mathrm{MeV}$, $a_{P}=9.82356 \mathrm{MeV}, \alpha=29.1048 \mathrm{MeV}$ and $\beta=12.1270 \mathrm{MeV}$. When ignoring the surface diffuseness in putting $\Delta=0$ in (28), the best-fit value are $a_{V}=15.6163 \mathrm{MeV}$, $a_{S}=17.9878 \mathrm{MeV}, a_{C}=0.692791 \mathrm{MeV}, a_{P}=10.8714 \mathrm{MeV}, \alpha=32.6655 \mathrm{MeV}$ and $\beta=13.6106 \mathrm{MeV}$.

We point out that the additional capacitance for asymmetry provided by the nuclear surface is associated with the drop of symmetry energy per nucleon with density. The ratio of the volume to surface symmetry coefficients can be used to assess the pace of that drop. We test the analytic result for the skin size within the Thomas-Fermi approximation and we use the results from microscopic mean-field calculations to constrain the drop in the symmetry energy at half of the normal density, $s=S\left(\rho_{0} / 2\right) / S\left(\rho_{0}\right)$, finding $0.57 \lesssim s \lesssim 0.83$.

For the collective asymmetry oscillations, we derive a simple boundary condition, following a consideration of the surface fed by the asymmetry flux from the nuclear interior. The nature of the condition depends on the nuclear mass, as the relative capacitance of the surface for asymmetry changes with the system size. As the size increases, the nature of the collective oscillation changes from a surface to a volume mode. 


\section{ACKNOWLEDGMENTS}

The hospitality extended by the Nuclear Theory Group of the Tohuku University, where this work was initiated, is gratefully acknowledged. Acknowledged is further a collaboration, on a topic related to the present work, with Sergio Souza and Betty Tsang [17]. Betty suggested an early version of Fig. 4. A discussion with Bill Lynch helped in clarifying an issue in Sec. II. Naftali Auerbach provided explanations on giant resonances. Stefan Typel and Michael Thoennessen helped in identifying literature. Dick Furnstahl generously supplied unpublished details of his calculations [7], employed in Figs. 15 and 16. A helpful discussion with Earle Lomon and a correspondence with Aurel Bulgac are further also acknowledged. This work was partially supported by the National Science Foundation under Grants PHY0070818, PHY-0245009 and INT-0124186. 


\section{REFERENCES}

[1] Isospin Physics in Heavy-Ion Collisions at Intermediate Energies, eds. B.-A. Li and U. Schröder, ISBN 1-56072-888-4 (Nova Science Publishers Inc., New York, 2001).

[2] J. M. Lattimer and M. Prakash, Astrophys. J. 550, 426 (2001).

[3] P. Danielewicz, R. Lacey and W. G. Lynch, Science 298, 1592 (2002) [Published online 31 October 2002; 10.1126/science.1078070]

[4] K. Oyamatsu, I. Tanihata, Y. Sugahara, K. Sumiyoshi and H. Toki, Nucl. Phys. A634, 3 (1998).

[5] B. A. Brown, Phys. Rev. Lett. 85, 5296 (2000).

[6] S. Typel and B. A. Brown, Phys. Rev. C 64, 027302 (2001).

[7] R. J. Furnstahl, Nucl. Phys. A706, 85 (2002).

[8] C. F. Weizsäcker, Zeit. f. Phys. 96, 431 (1935).

[9] H. A. Bethe and R. F. Bacher, Rev. Mod. Phys. 8, 82 (1936).

[10] W. D. Myers and W. J. Swiatecki, Nucl. Phys. A81, 1 (1966).

[11] M. A. Preston and R. K. Bhaduri, Structure of the Nucleus, Addison-Wesley, Reading, 1975.

[12] P. Möller, J. R. Nix, W. D. Myers and W. J. Swiatecki, Atomic Data and Nuclear Data Tables 59, 185 (1995).

[13] K. Pomorski and J. Dudek, nucl-th/020501, 2002.

[14] W. D. Myers and W. J. Swiatecki, Ann. of Phys. 55, 395 (1969).

[15] W. D. Myers and W. J. Swiatecki, Ann. of Phys. 84, 186 (1974).

[16] W. D. Myers, Droplet Model of Atomic Nuclei, IFI/Plenum, New York, 1975.

[17] S. R. Souza et al., Phys. Rev. C 67, 051602 (2003).

[18] J. W. Gibbs, The Collected Works, Yale Univ. Press, New Haven, 1948.

[19] G. Audi and A. H. Wapstra, Nucl. Phys. A595, 409 (1995), http://www.nndc.bnl.gov/nndcsr/masses

[20] L. Ray, Phys. Rev. C 19, 1855 (1979).

[21] S. Shlomo and R. Schaeffer, Phys. Lett. B 83, 5 (1979).

[22] C. J. Batty, E. Friedman, H. J. Gils and H. Rebel, Advances in Nuclear Physics, vol. 19, ed. J. W. Negele, (Plenum Press, New York, 1989) p. 1, and refs. therein.

[23] W. R. Gibbs and J.-P. Dedoner, Phys. Rev. C 46, 1825 (1992).

[24] V. E. Starodubsky and N. M. Hintz, Phys. Rev. C 49, 2118 (1994).

[25] T. Suzuki et al., Phys. Rev. Lett. 75, 3241 (1995).

[26] S. Karataglidis, K. Amos, B. A. Brown and P. K. Deb, Phys. Rev. C 65, 044306 (2002).

[27] B. C. Clark, L. J. Kerr and S. Hama, Phys. Rev. C 67, 054605 (2003).

[28] A. Bohr and B. R. Mottelson, Nuclear Structure (Benjamin, New York), Vol. I (1969) and II (1975).

[29] M. Goldhaber and E. Teller, Phys. Rev. 74, 1046 (1948).

[30] H. Steinwedel and H. Jensen, Z. Naturforschung 5A, 413 (1950).

[31] W. D. Myers, W. J. Swiatecki and C. S. Wang, Nucl. Phys. A436, 185 (1985).

[32] W. D. Myers and W. J. Swiatecki, Phys. Rev. C 63, 034318 (2001).

[33] C. W. de Jager, H. de Vries, and C. de Vries, Atomic Data and Nuclear Data Tables 14,479 (1974).

[34] A. Tarantola, Inverse Problem Theory, Methods for Data Fitting and Model Parameter Estimation, Elsevier, Amsterdam, 1987. 
[35] A. N. Tikhonov, Sov. Math. Dokl. 4, 1035 (1963).

[36] C. J. Horowitz and J. Piekarewicz, Phys. Rev. Lett. 86, 5647 (2001); Phys. Rev. C 64, 064616 (2001); Phys. Rev. C 66, 055803 (2002).

[37] S. Mizutori, J. Dobaczewski, G. A. Lalazissis, W. Nazarewicz and P. G. Reinhard, Phys. Rev. C 61, 044326 (2000).

[38] D. Vretenar, P. Finelli, A. Ventura, G. A. Lalazissis and P. Ring, Phys. Rev. C 61, 064307 (2000).

[39] B. Nerlo-Pomorska and K. Mazurek, Phys. Rev. C 66, 064305 (2002).

[40] C. J. Horowitz, S. J. Pollock, P. A. Souder and R. Michaels, Phys. Rev. C 63, 025501 (2001).

[41] I. Tanihata et al., Phys. Lett. B 289, 261 (1992).

[42] B. A. Brown and W. A. Richter, Phys. Rev. C 54, 673 (1996).

[43] S. Goriely, M. Samyn, P.-H. Heenen, J. M. Pearson and F. Tondeur, Phys. Rev. C 66, 024326 (2002).

[44] H. Müller and B. D. Serot, Phys. Rev. C 522072 (1995).

[45] L. Engvik, M. Hjorth-Jensen, R. Machleidt, H. Müther and A. Polls, Nucl. Phys. A627, 85 (1997).

[46] A. Akmal, V. R. Pandharipande and D. G. Ravenhall, Phys. Rev. C 58, 1804 (1998).

[47] S. S. Dietrich and B. L. Berman, Atomic Data and Nuclear Data Tables 38, 199 (1988).

[48] R. Pitthan et al., Phys. Rev. C 19, 1251 (1979).

[49] P. Ring and P. Schuck, The Nuclear Many-Body Problem (Springer-Verlag, New York, 1980).

[50] W. D. Myers, W. J. Swiatecki, T. Kodama, L. J. El-Jaick and E. R. Hilf, Phys. Rev. C 15, 2032 (1977).

[51] D. Pines and Ph. Nozieres, The Theory of Quantum Liquids (Benjamin, New York, 1966).

[52] S. Khamerdzhiev, J. Speth and G. Tertychny, Nucl. Phys. A624, 328 (1997).

[53] A. R. Bodmer and Q. N. Usmani, Phys. Rev. C 67, 034305 (2003).

[54] P. Danielewicz, Nucl. Phys. A673, 375 (2000). 


\section{FIGURES}

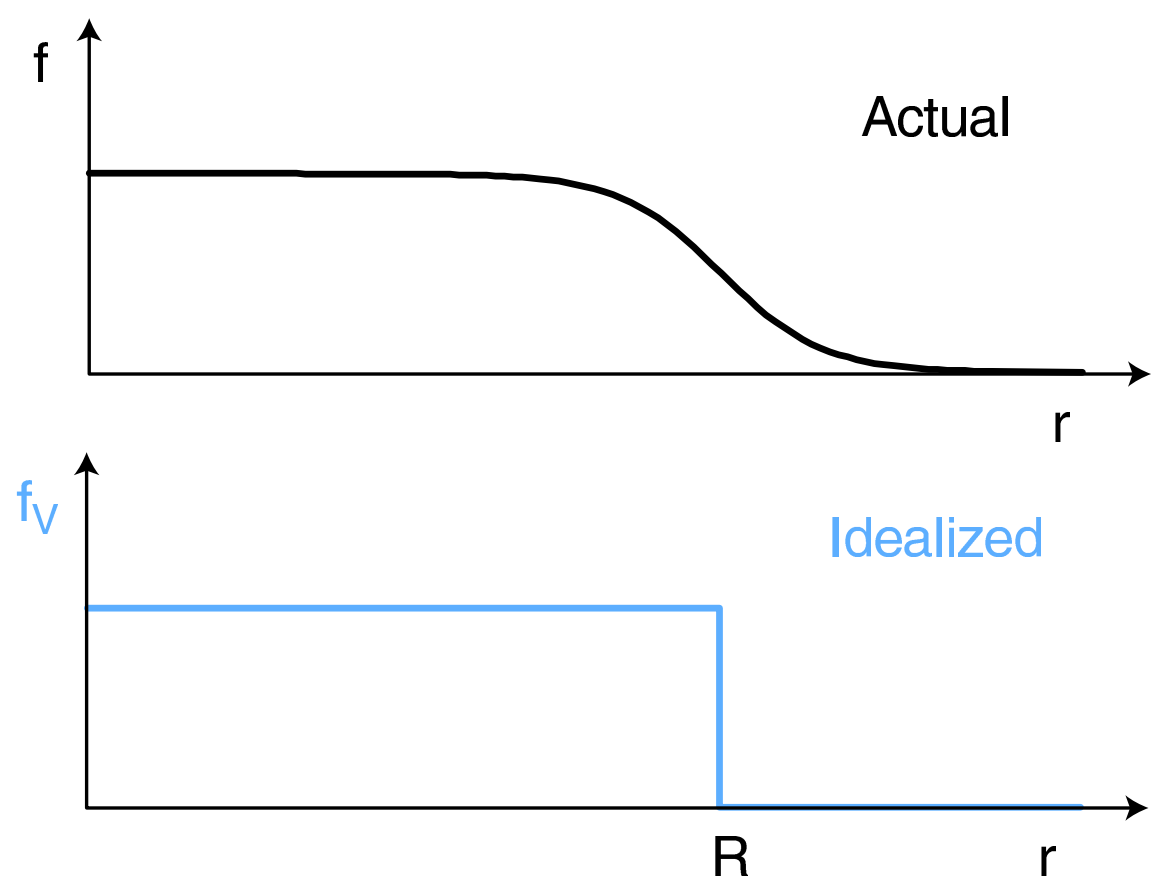

FIG. 1. Illustration for the construction of surface quantities following Gibbs [18]. The top shows the variation of the density $f$ for some quantity $F$ within the system. The bottom shows an idealized reference system, where the density changes in a discontinuous manner, right at the surface, between the density values $f_{V}$ characteristic for the system away from the interface. The surface quantities $F_{S}$ are defined as the difference between $F$, for a macroscopic volume containing the interface, and $F_{i d}$, for the same volume in the reference system, $F_{S}=F-F_{i d}$. 


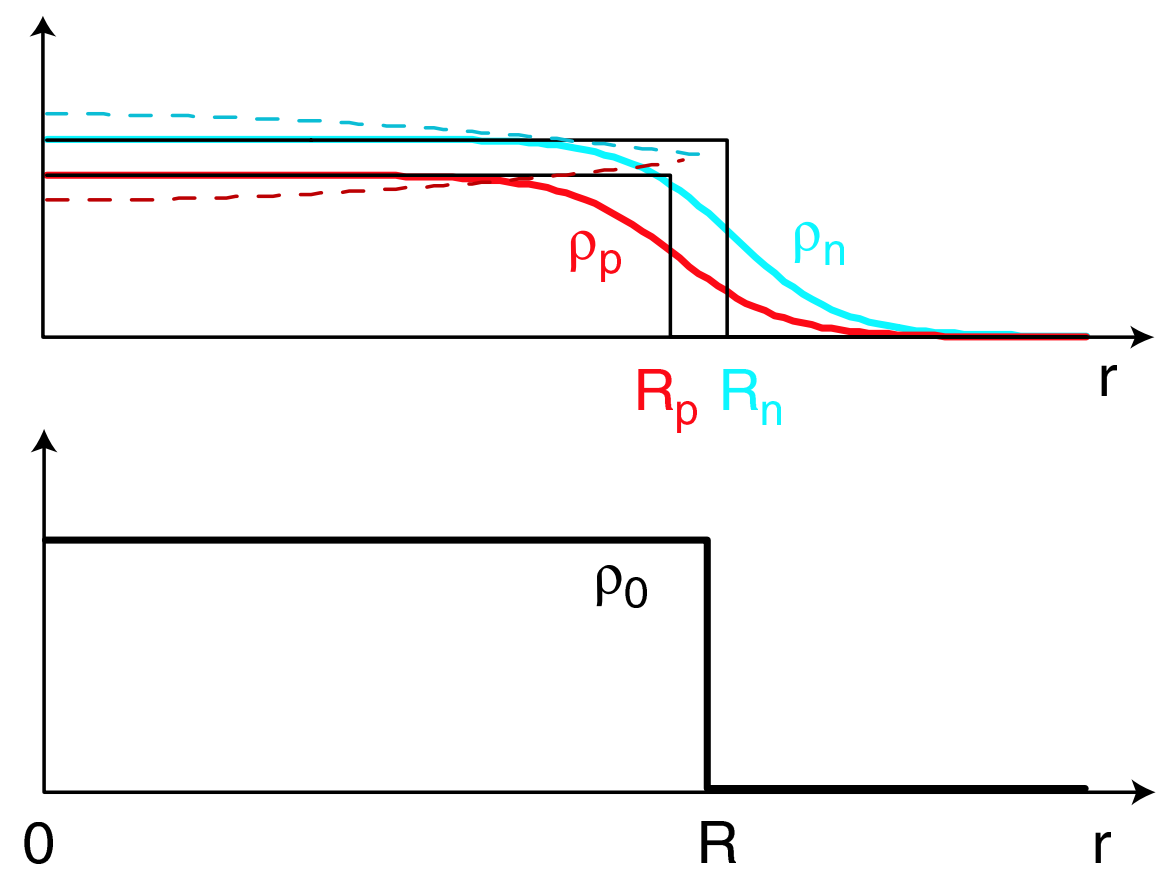

FIG. 2. In a two-component system, the surface-attributable particle-asymmetry can arise even when the surface is constructed to have no net particle number. For a spherical ground-state nucleus, this corresponds to the situation when the sharp-sphere equivalent proton $R_{p}$ and neutron $R_{n}$ radii differ from each other and bracket the matter sharp-sphere radius $R$. The dashed lines in the top part of the figure illustrate the polarization of a nuclear interior, in the form of a sharp-edged sphere of radius $R$, by the Coulomb interactions. 


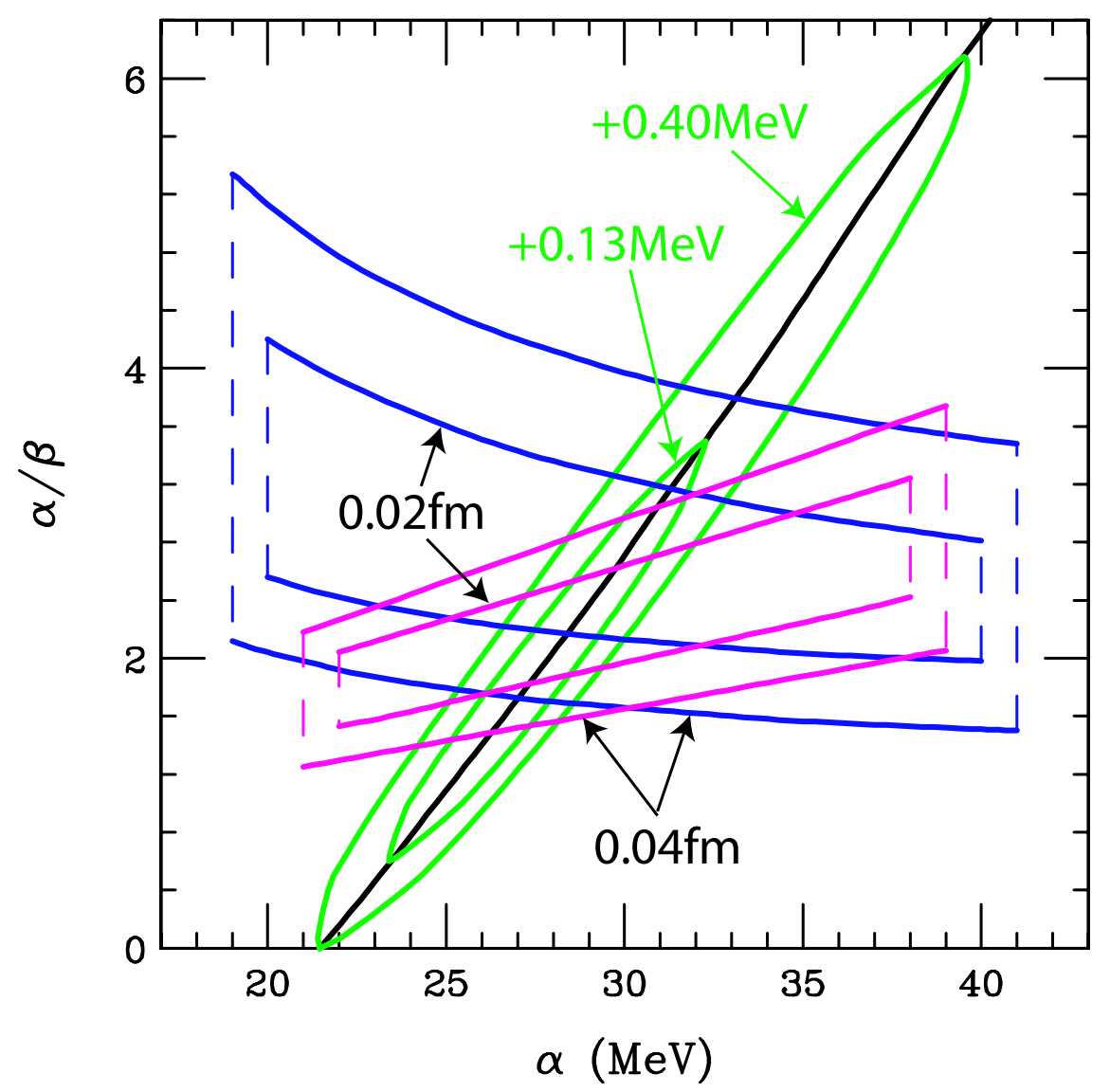

FIG. 3. Results from optimizations of the volume parameter $\alpha$ and the volume-to-surface parameter ratio $\alpha / \beta$. The thick solid diagonal line shows the optimal values of $\alpha$ at fixed $\alpha / \beta$ when fitting the nuclear masses. The oval contour lines show the loci of parameter combinations that yield average absolute deviations from the measured nuclear masses larger by 0.13 and $0.40 \mathrm{MeV}$, respectively, than a best-fit parameter combination. The rectangular contour lines indicate the loci of parameter combinations at constant $\chi^{2}$ when fitting the asymmetry skins deduced from data, either following Eq. (37) or (40). Only the solid more horizontal portions of the skin-thickness contours are meaningful - the vertical dashed portions serve only to guide the eye. The loci are for the $\chi^{2}$-values assuming a combination of the standard statistical deviation and of the average systematic error of a skin-size formula of 0.02 and $0.04 \mathrm{fm}$, respectively, with the solid positively sloped contours representing Eq. (40) and the negatively sloped representing Eq. (37). 


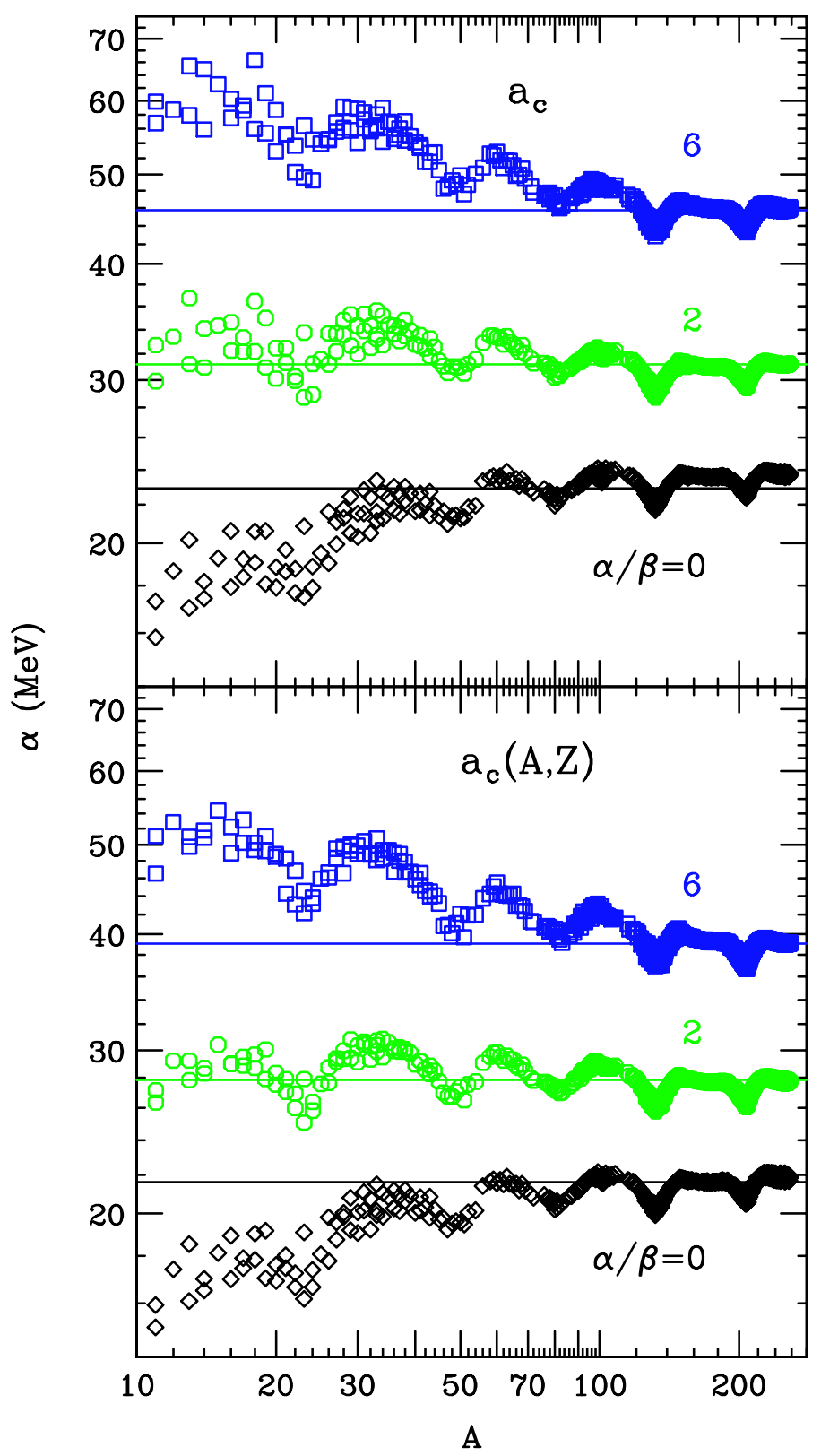

FIG. 4. Values of the volume symmetry parameter $\alpha$ from inverting the energy formula at different values of $\alpha / \beta$, for individual nuclei with $|N-Z| / A>0.2$, as a function of $A$. The top panel shows results for the energy formula with $\Delta=0$ and the bottom for the formula with $\Delta$ given by Eq. (29). The different symbols represent results from the inversions for $\alpha / \beta=6$ (squares), 2 (circles) and 0 (diamonds), respectively. The horizontal lines represent values of $\alpha$ from the global fits at the different $\alpha / \beta$. 


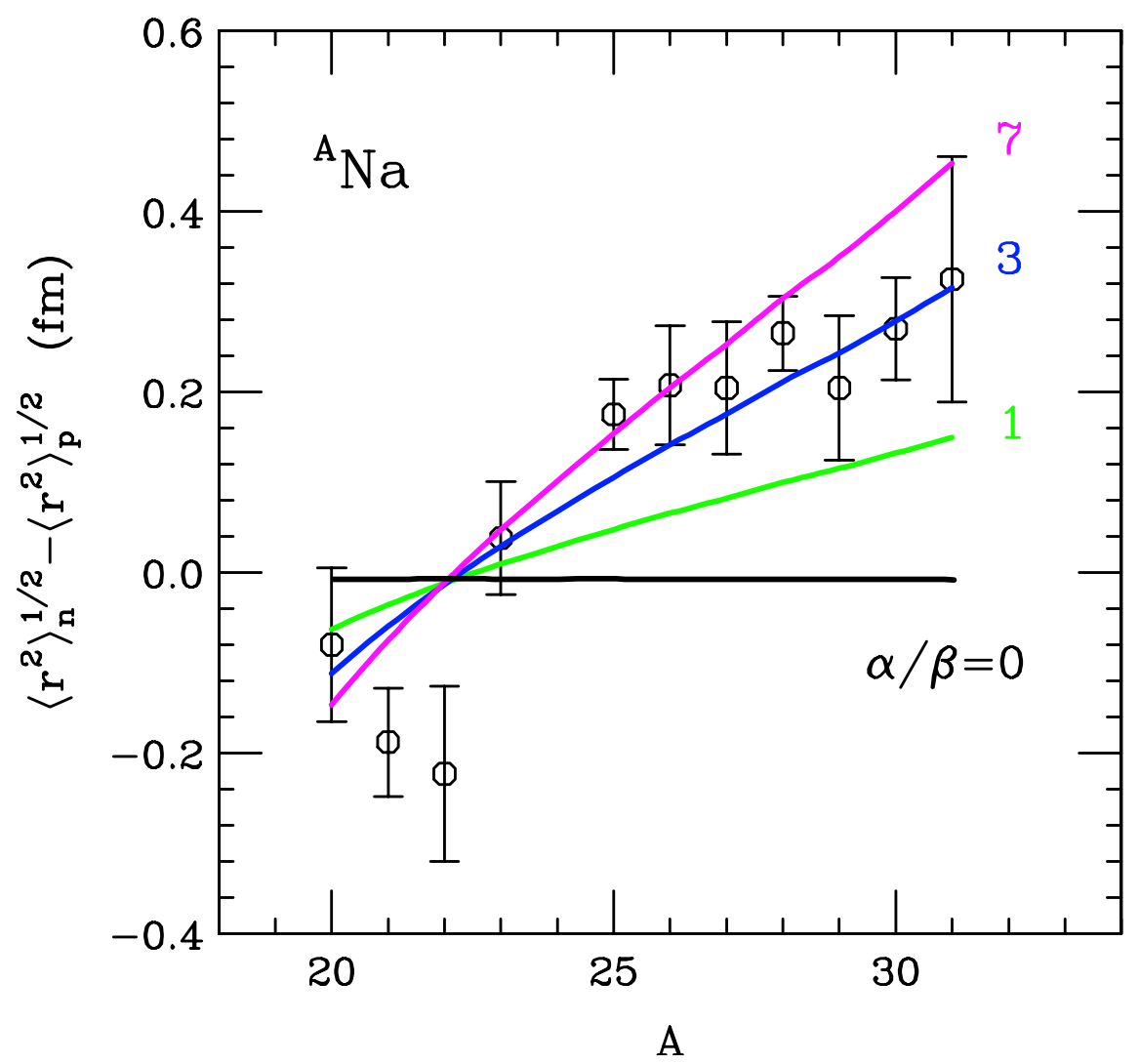

FIG. 5. Difference between the neutron and proton $\mathrm{rms}$ radii for $\mathrm{Na}$ isotopes as a function of the mass number. The symbols represent results from the data analysis Ref. [25]. The different lines represent results from Eq. (37) for the indicated values of $\alpha / \beta, d_{n}-d_{p}=0$ and $\alpha$ taken from the binding-energy correlation valley in Fig. 3. 


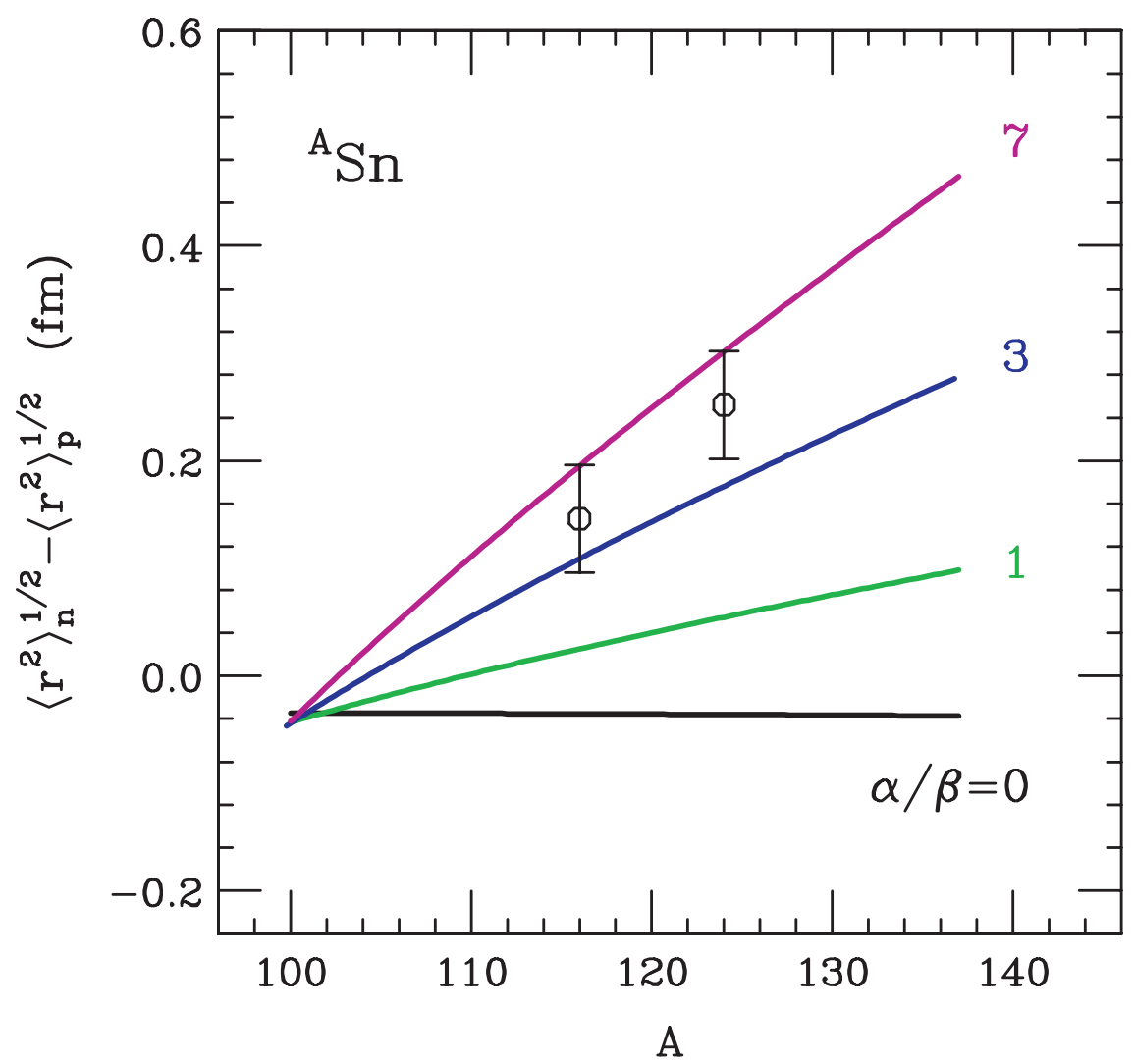

FIG. 6. Difference between the neutron and proton rms radii for Sn isotopes as a function of the mass number. The symbols represent results from the data analysis of Ref. [20]. The different lines represent results from Eq. (37) for the indicated values of $\alpha / \beta, d_{n}-d_{p}=0$ and $\alpha$ taken from the binding-energy correlation valley in Fig. 3. 


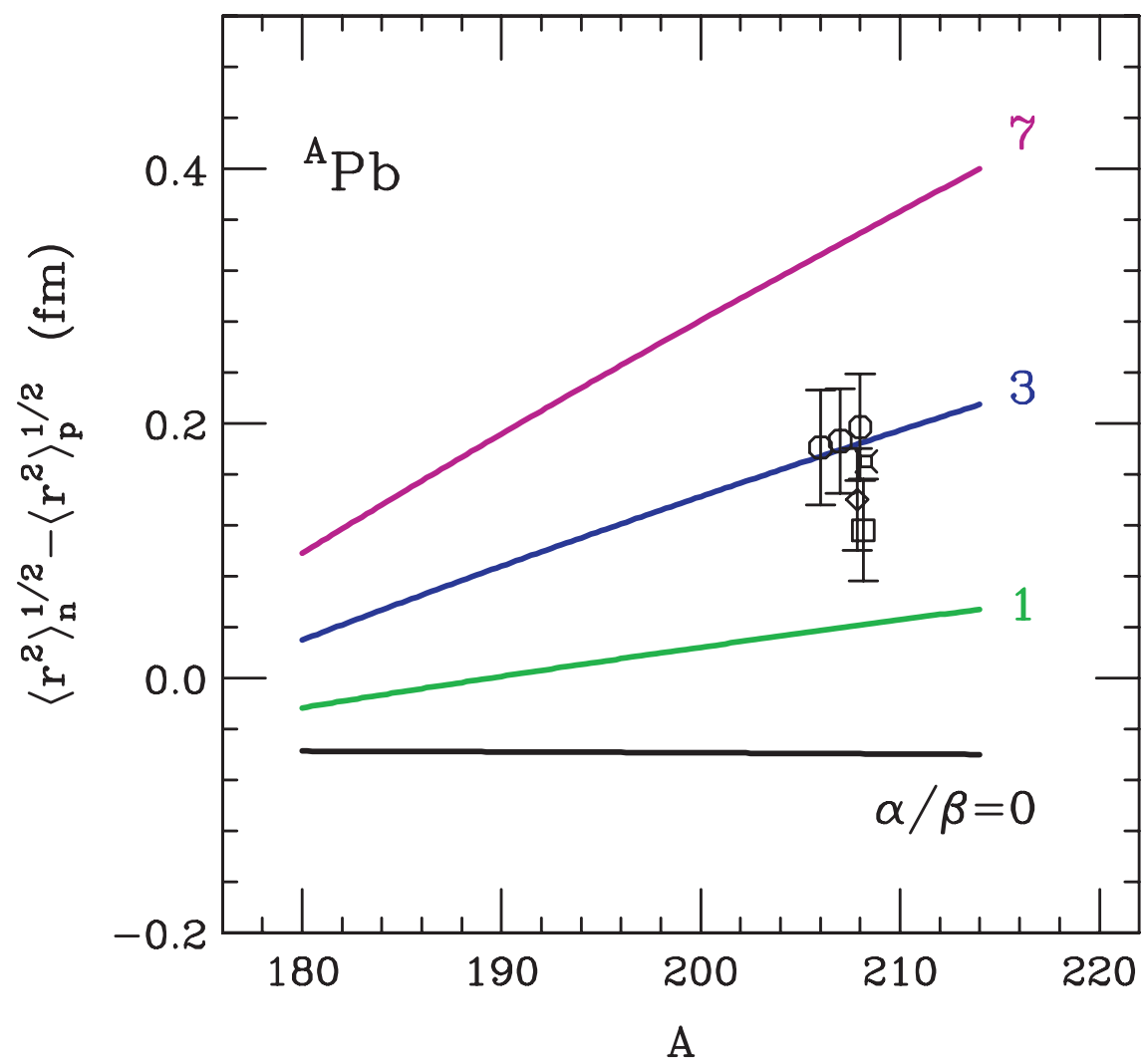

FIG. 7. Difference between the neutron and proton rms radii for $\mathrm{Pb}$ isotopes as a function of the mass number. The symbols represent results from the data analysis of Refs. [24] (circles), [20] (a diamond), [27] (a square) and [26] (a cross). The different lines represent results from Eq. (37) for the indicated values of $\alpha / \beta, d_{n}-d_{p}=0$ and $\alpha$ taken from the binding-energy correlation valley in Fig. 3. 


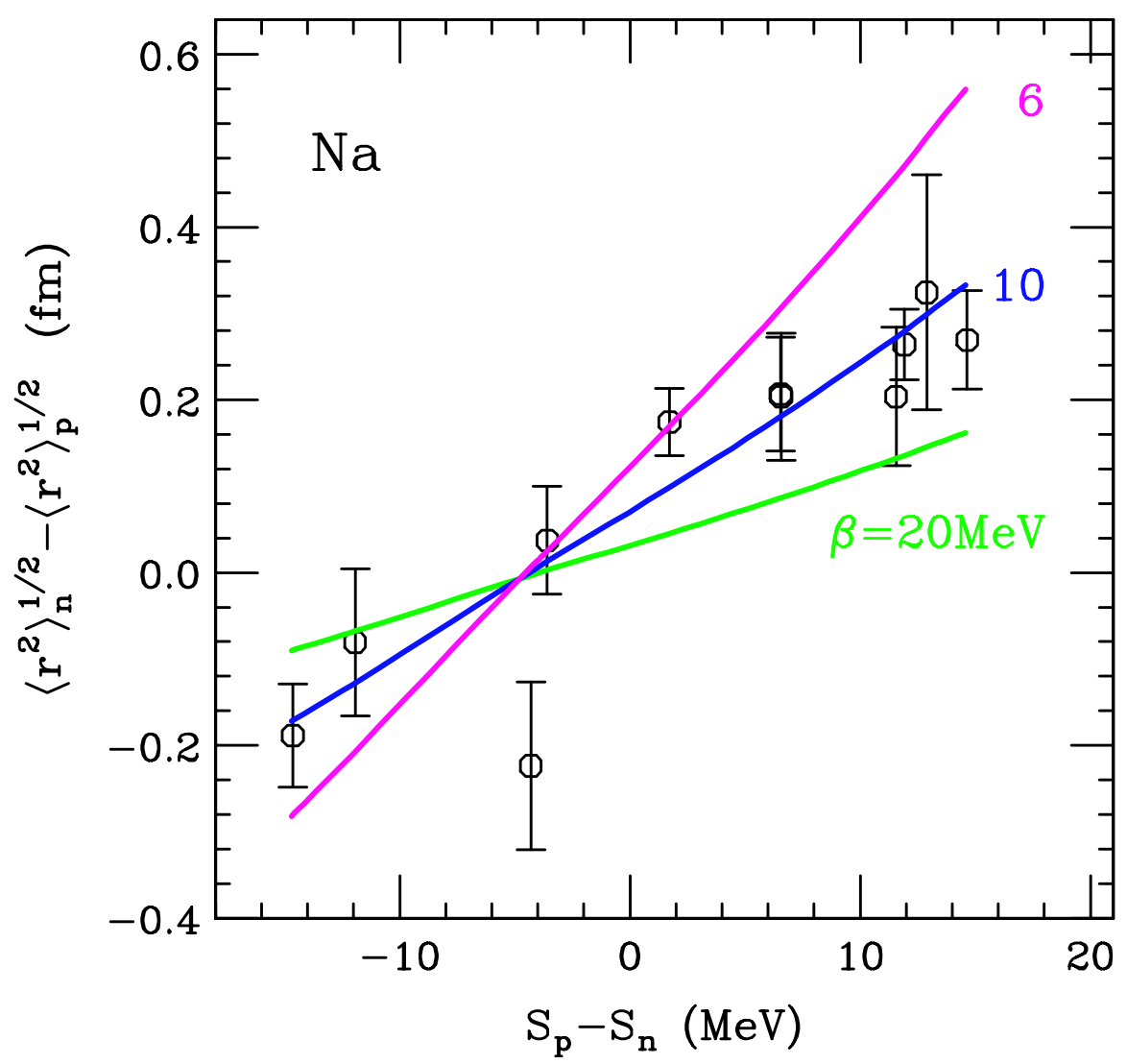

FIG. 8. Difference between the neutron and proton rms radii vs the difference between the proton and neutron separation energies for Na isotopes. The symbols represent data of Ref. [25]. The lines represent predictions of Eq. (40) for different indicated values of $\beta$ (in MeV) with the values of $\alpha$ made to follow the binding-energy correlation in Fig. 3. 


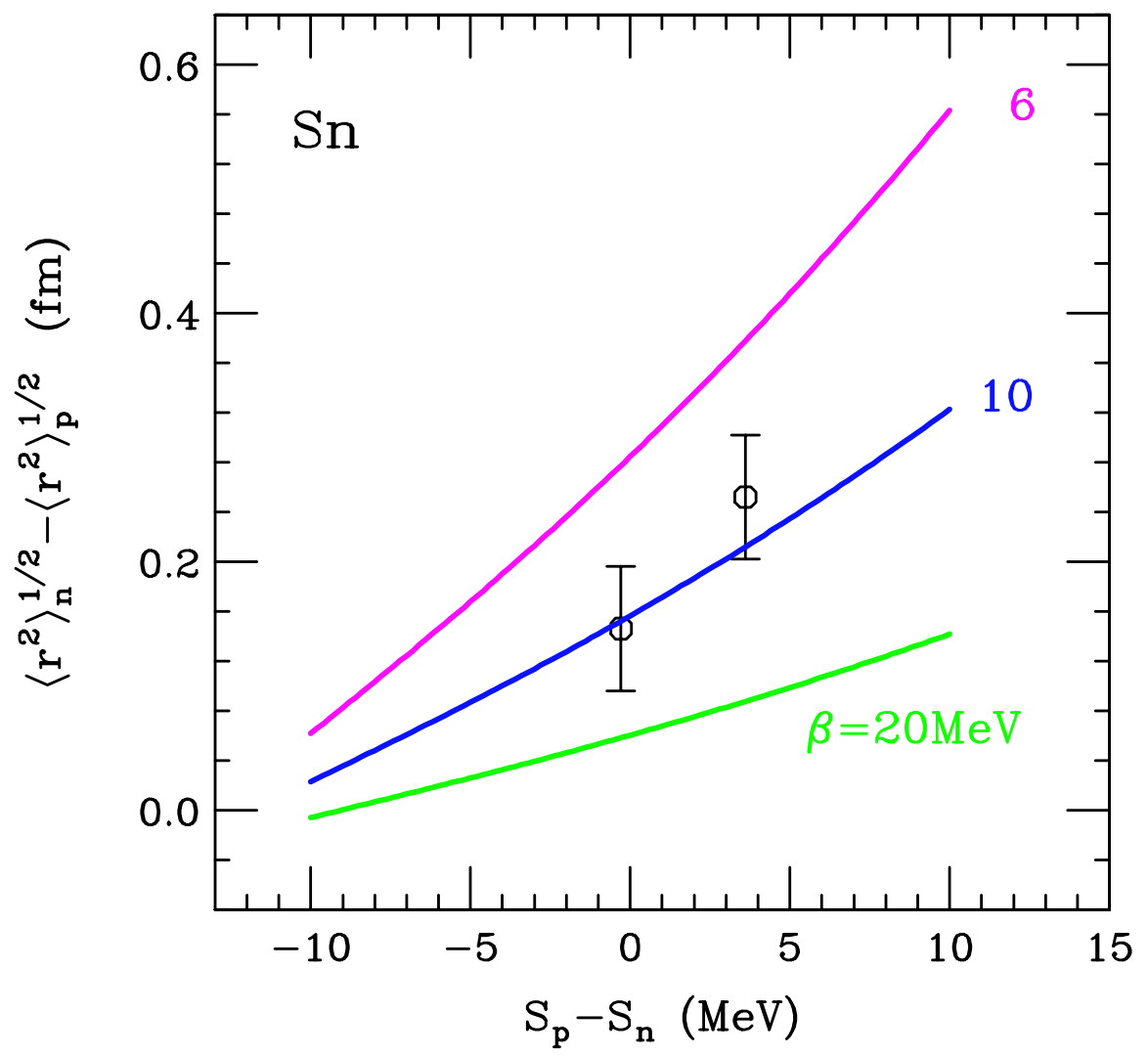

FIG. 9. Difference between the neutron and proton rms radii vs the difference between the proton and neutron separation energies for Sn isotopes. The symbols represent data of Ref. [20]. The lines represent predictions of Eq. (40) for different indicated values of $\beta$ (in MeV) with the values of $\alpha$ made to follow the binding-energy correlation in Fig. 3. 


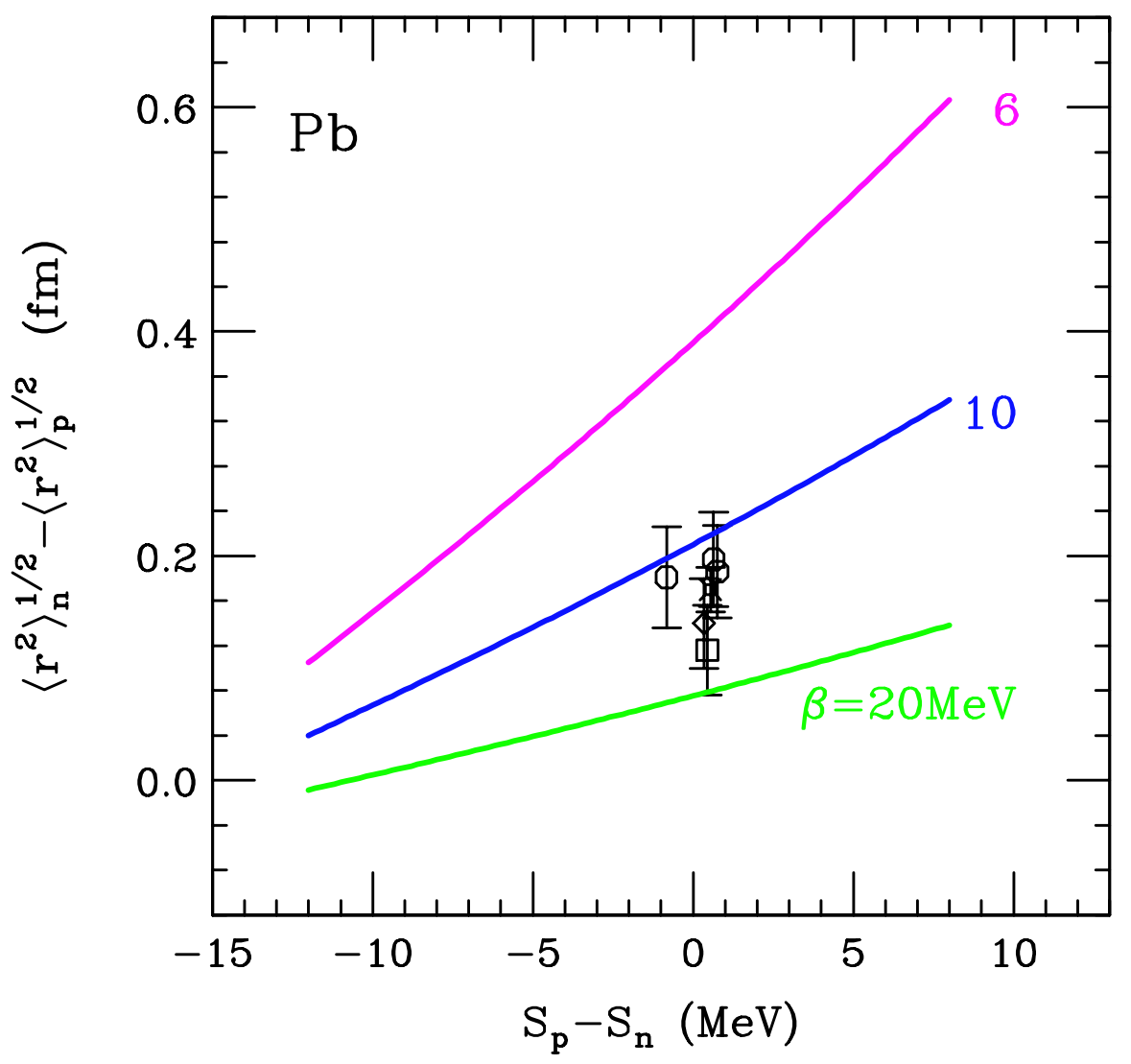

FIG. 10. Difference between the neutron and proton rms radii vs the difference between the proton and neutron separation energies for $\mathrm{Pb}$ isotopes. The symbols represent results from the data analysis of Refs. [24] (circles), [20] (a diamond), [27] (a square) and [26] (a cross). The lines represent predictions of Eq. (40) for different indicated values of $\beta$ (in $\mathrm{MeV}$ ) with the values of $\alpha$ made to follow the binding-energy correlation in Fig. 3. 


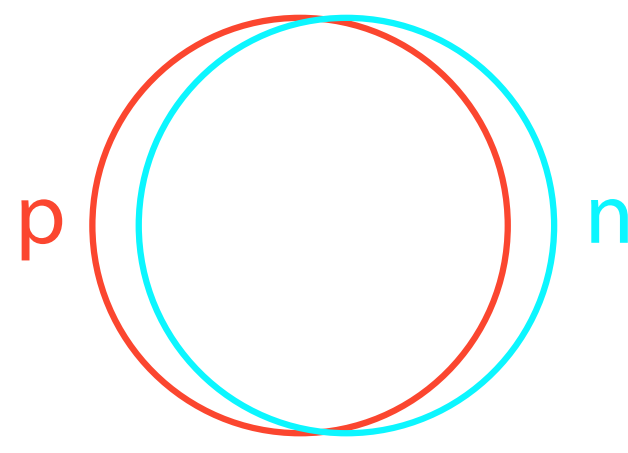

GT

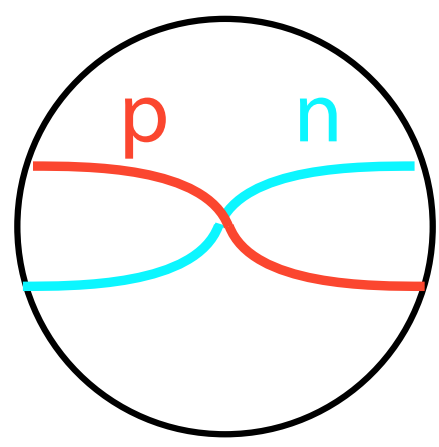

SJ

FIG. 11. In the GT model [29] of the GDR, neutron and proton distributions are assumed to oscillate, unchanged with respect to their centers, against each other. In the SJ model $[29,30]$ of the GDR, particle asymmetry oscillates within the nuclear interior, with the flux of asymmetry vanishing at the nuclear surface. 


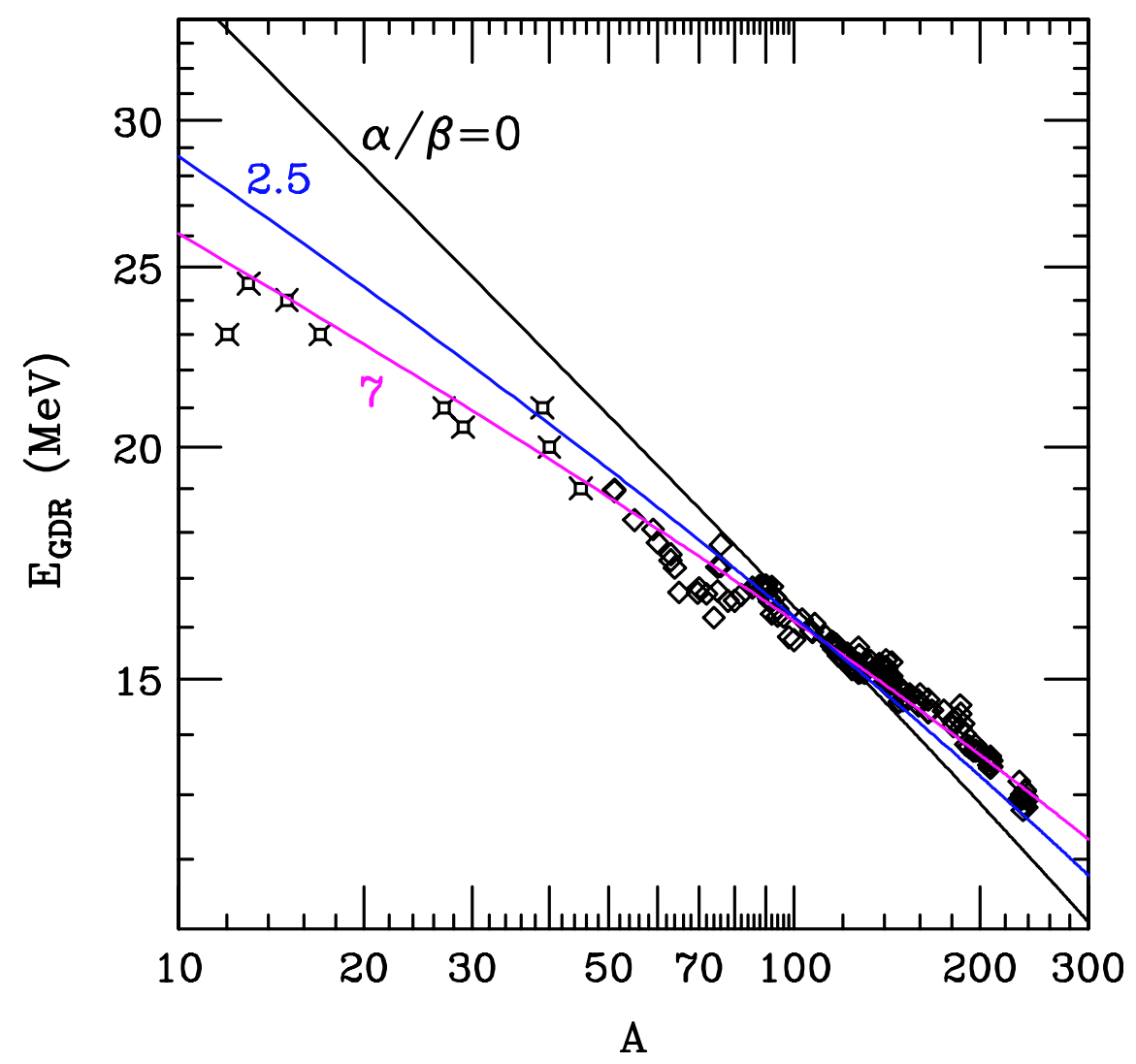

FIG. 12. Energy of giant dipole resonance as a function of mass number $A$. Symbols represent photoabsorption data from the compilation [47]. For mass numbers $A>50$, we use the energies from the Lorentzian function fits from [47]. When a splitting characteristic for a deformed nucleus is present, we plot the equivalent energy for a spherical nucleus. For mass numbers $A<50$, we plot the peak values for nuclei for which a clear isolated peak is present in the photoabsorption cross section [47]. The lines represent energies calculated from $E_{G D R}=(2 \sqrt{(N Z)} / A) \hbar c_{a} q_{11}$ with $c_{a}$ being a fit parameter and with $q_{11}$ obtained from (57) at the different indicated values of $\alpha / \beta$. 


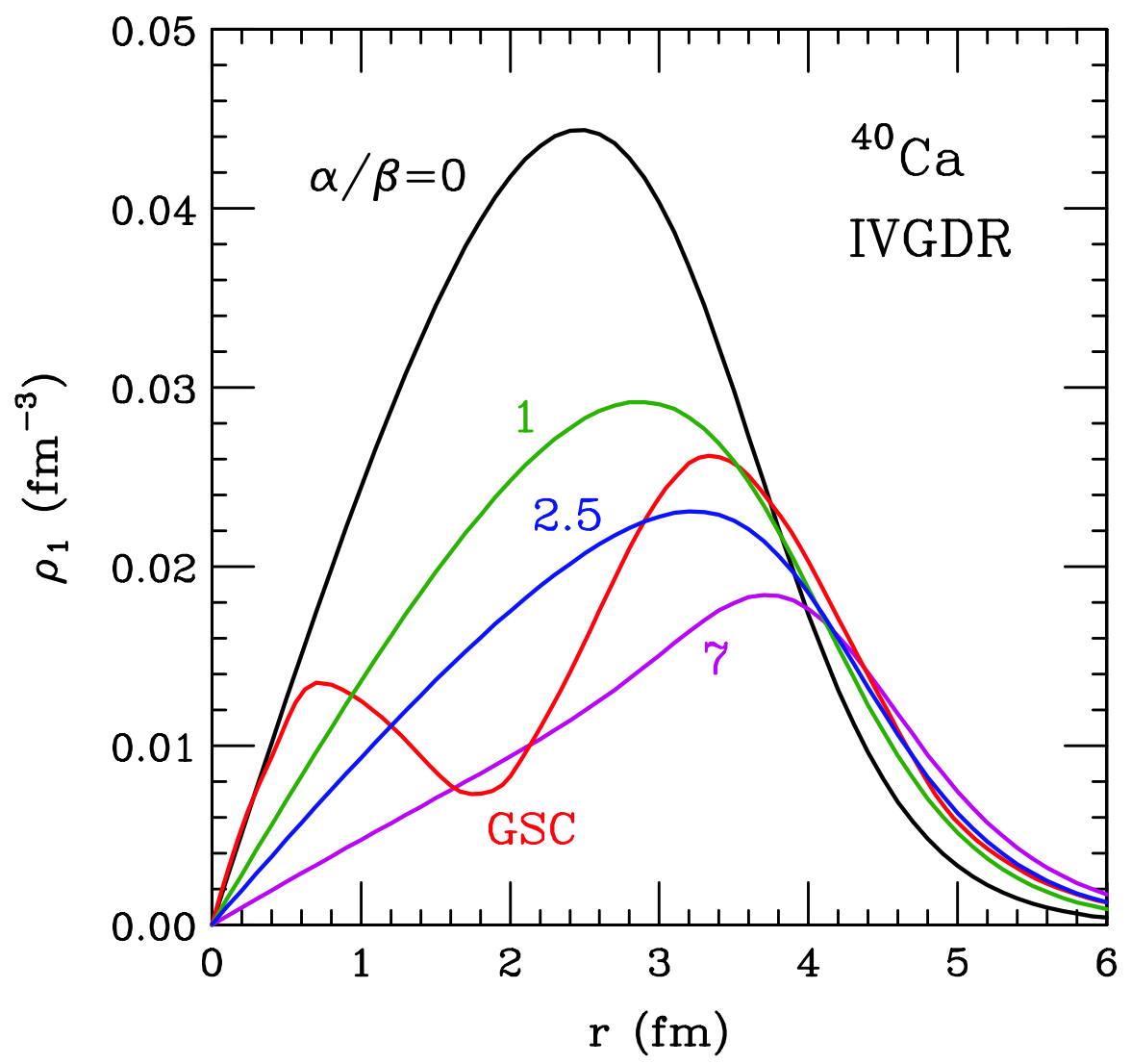

FIG. 13. GDR transition density $\rho_{1}$ for ${ }^{40} \mathrm{Ca}$, as a function of the distance $r$ from nuclear center. The lines marked with the respective values of parameter ratio $\alpha / \beta$ represent the densities obtained from Eq. (62). The line marked GSC represents density from the microscopic calculations of Ref. [52], including effects of the $2 \mathrm{p}-2 \mathrm{~h}$ excitations and ground-state correlations. 


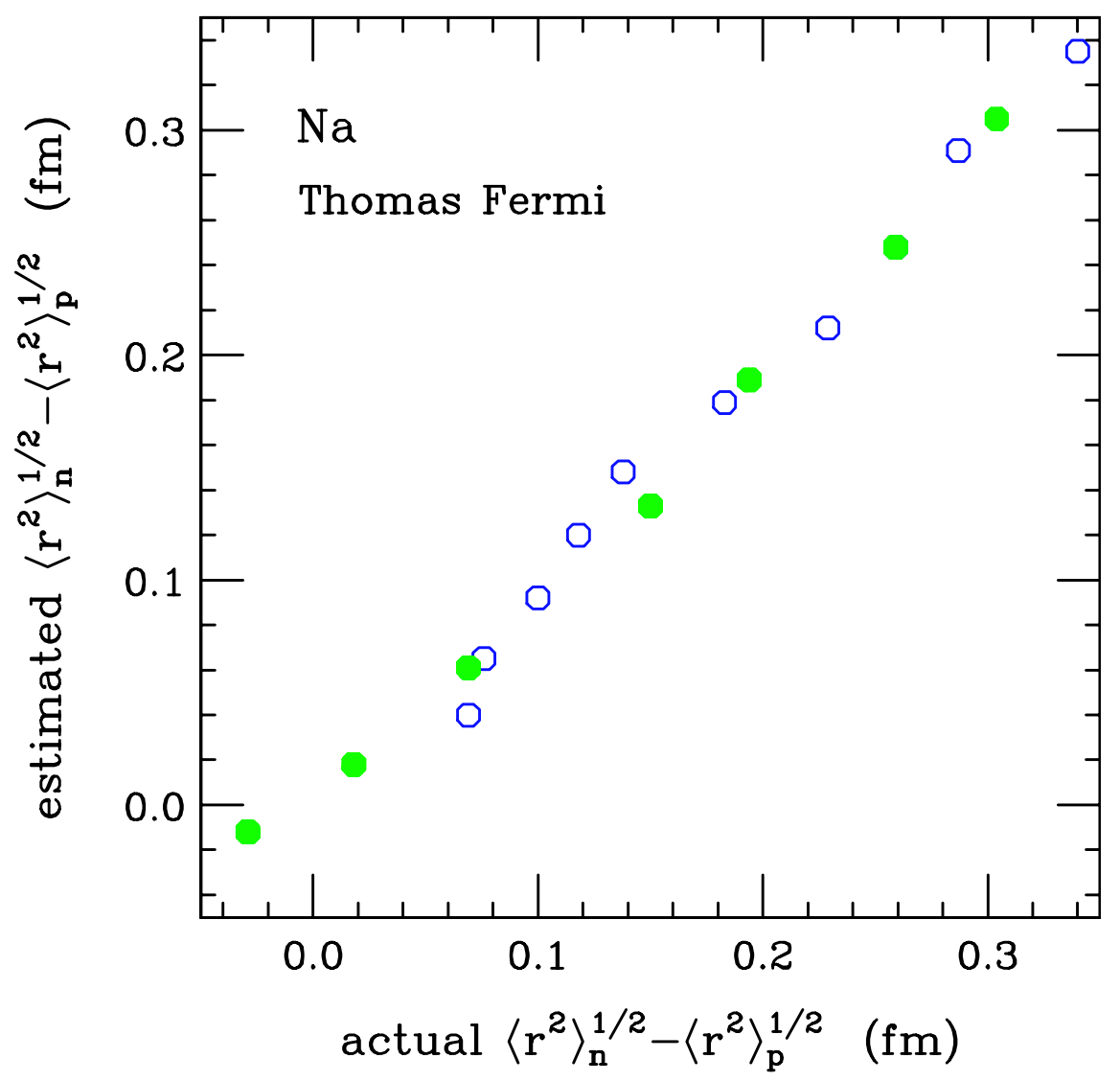

FIG. 14. Size of the asymmetry skin estimated with Eq. (37) vs size obtained directly from the calculated nucleon distributions, within the Thomas-Fermi calculations of sodium isotopes. The filled circles represent the results for $\alpha / \beta=3$, while changing the isotope mass. The open circles represent the results for ${ }^{30} \mathrm{Na}$, when changing the values of $\alpha / \beta$ and correlating the changes in $\alpha$ to follow the valley in Fig. 3 . 


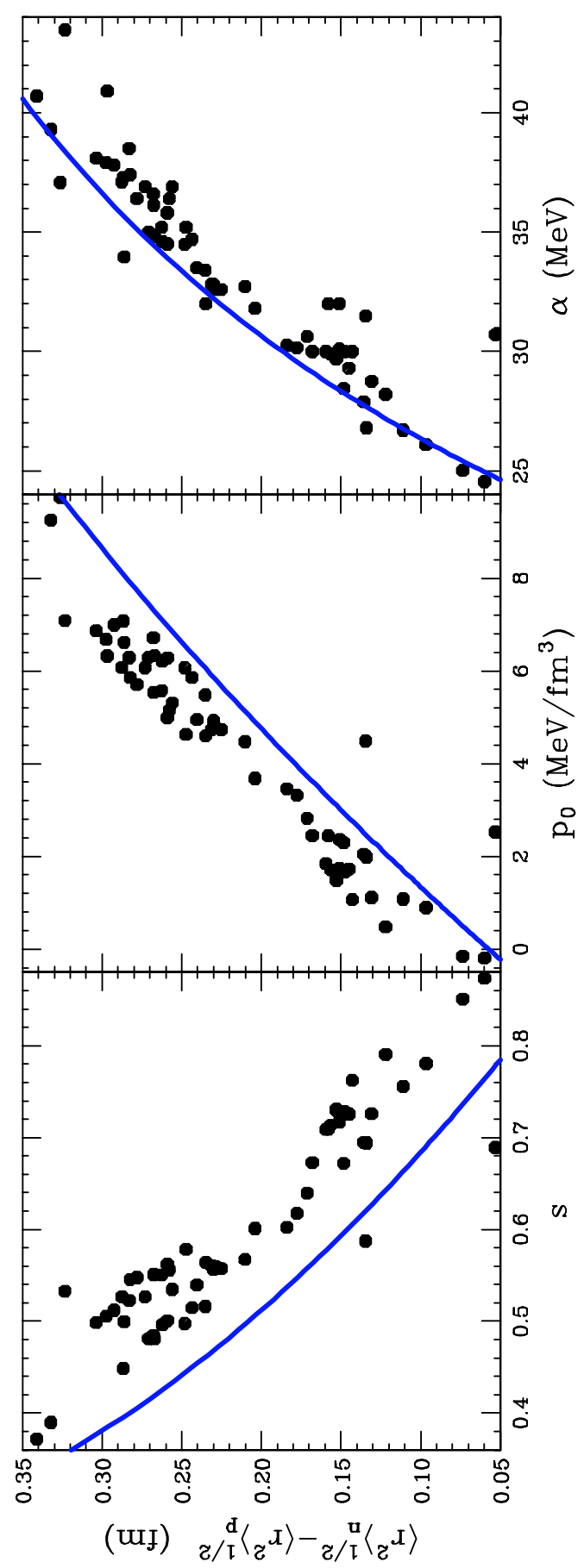

FIG. 15. Size of the asymmetry skin in models vs the reduction factor $s$ for the symmetry energy at $\rho_{0} / 2$ (left panel), vs the scaled derivative $[4,7,53]$ of the energy $p_{0}=\rho_{0}^{2}(d S / d \rho)$ at $\rho_{0}$ (center panel) and vs the volume symmetry parameter $\alpha$ (right panel). Symbols indicate results for the variety of mean-field models explored by Furnstahl [7]. Lines indicate Thomas-Fermi results, from Eq. (37) using (72). 


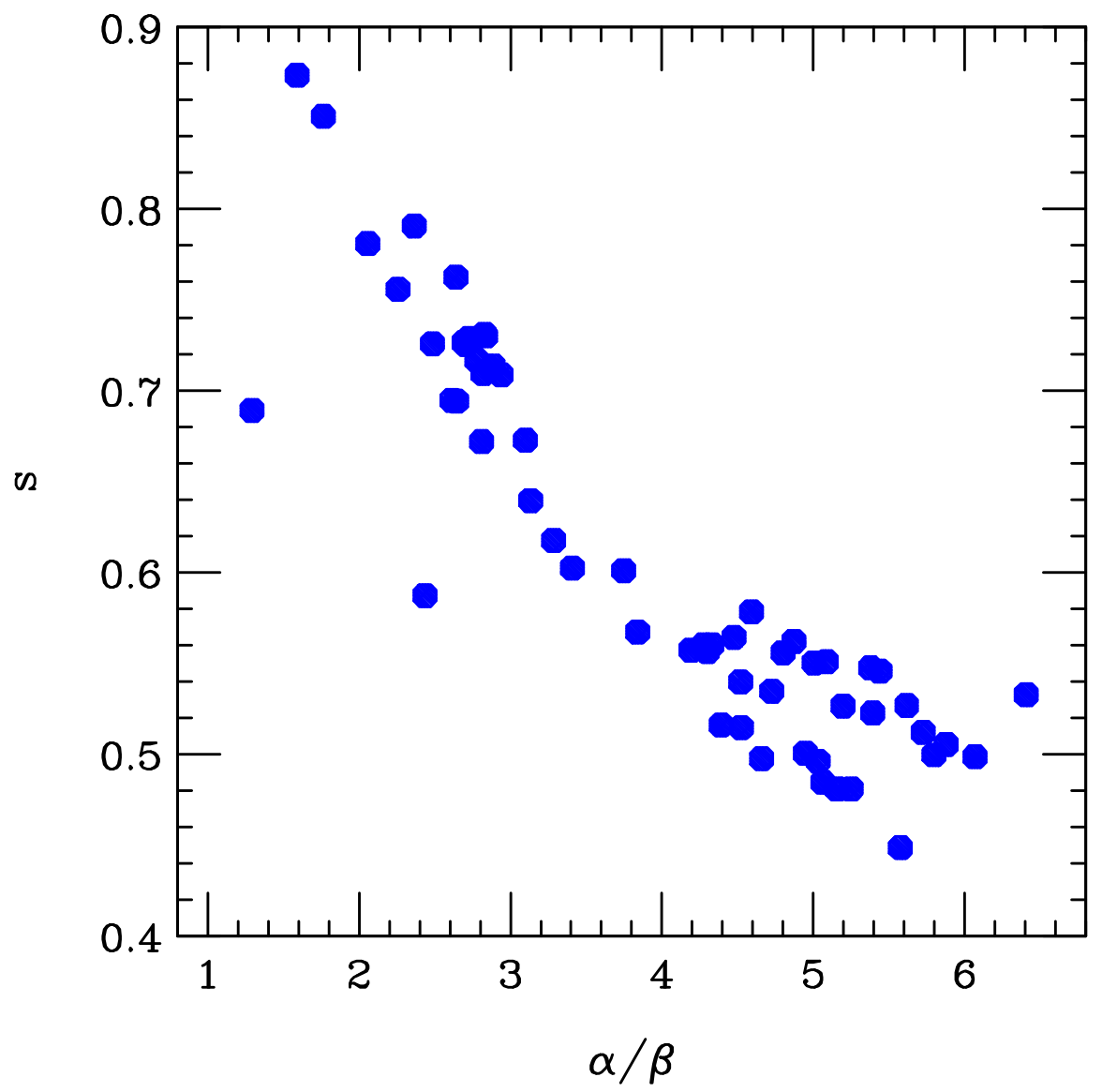

FIG. 16. Reduction factor $s$ for the symmetry energy per nucleon at $\rho_{0} / 2$ vs the symmetry parameter ratio $\alpha / \beta$ deduced for the mean-field models from the calculated [7] ${ }^{208} \mathrm{~Pb}$ skins and Eq. (37). 